\title{
Fermionic phases and their transitions induced by competing finite-range interactions
}

\author{
M. Szyniszewski ${ }^{1,}$ * and H. Schomerus ${ }^{1}$ \\ ${ }^{1}$ Department of Physics, Lancaster University, Lancaster LA1 4 YB, United Kingdom
}

(Dated: August 8, 2018)

\begin{abstract}
We identify ground states of one-dimensional fermionic systems subject to competing repulsive interactions of finite range, and provide phenomenological and fundamental signatures of these phases and their transitions. Commensurable particle densities admit multiple competing charge-ordered insulating states with various periodicities and internal structure. Our reference point are systems with interaction range $p=2$, where phase transitions between these charge-ordered configurations are known to be mediated by liquid and bond-ordered phases. For increased interaction range $p=4$, we find that the phase transitions can also appear to be abrupt, as well as being mediated by reemergent ordered phases that cross over into liquid behavior. These considerations are underpinned by a classification of the competing charge-ordered states in the atomic limit for varying interaction range at the principal commensurable particle densities. We also consider the effects of disorder, leading to fragmentization of the ordered phases and localization of the liquid phases.
\end{abstract}

\section{INTRODUCTION}

One-dimensional fermionic physics substantially differs from its higher-dimensional counterparts. Usual descriptions of interactions, such as Fermi liquid theory, break down, $\frac{112}{12}$ which results in an absence of quasiparticle excitations in the system. Under many circumstances, the appropriate theory describing these systems

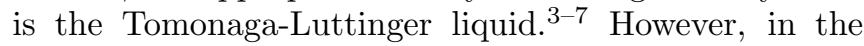
presence of repulsive interactions and at commensurable particle densities the system can form charge-ordered phases $\frac{18 \sqrt{12}}{1 n}$ which it displays insulating properties.

The nature of the quantum phase transition between liquid and charge-ordered phases has been uncovered in detailed theoretical studies 13 The main factor that drives the phase transition is the competition between the kinetic energy and the interaction terms that can order the system. In particular, lattice fermion models exhibit an interplay of short-range kinetic quantum fluctuations arising from the uncertainty principle and repulsive finite-range interactions that cause the insulating phases $\frac{18}{21}$ These studies have revealed various transition scenarios, including the emergence of a 'strange metallic phase' that was later identified to be of bond order ${ }^{22} 27$ - a dimerized phase with alternating bond strengths and without charge ordering. To determine these quantum phase transitions precisely, various methods have been proposed, such as investigation of the ground-state curvature, $\frac{19}{19}$ structure factors, 20127 bond entropy ${ }^{28}$ and scaling of the gap $\sqrt[20]{20}$ Decaying behaviour of the correlation functions was also found to be a distinguishing feature for the aforementioned quantum phases. $\frac{27 / 29}{2}$ Since spinless fermions in one dimension are equivalent to hard-core bosons,, $30 \mid 33$ these observations are also of great interest for analogous bosonic systems ${ }^{34}$ Recent advancements in optical lattices have indeed made it possible to engineer condensed matter systems $\frac{35136}{3}$ that allow to directly observe the liquid-toinsulator transition. 37 [39

Overall, however, the understanding of these transi- tions is still restricted to a small number of relatively simple and mutually well compatible charge-ordered states. As the range of interactions increases, the variety of competing charge-ordered states increases rapidly. This situation raises a number of unresolved questions. On one hand, the transitions between these phases may proliferate as well, and could squeeze out the insulating behavior. On the other hand, the liquid phases could be suppressed depending on the complexity of the charge configurations of competing states. Moreover, the bond-ordered phases may survive the introduction of additional interactions, or, completely new transition scenarios could arise.

In this paper, we address these questions within a model that exhibits multiple transitions between a variety of ordered states of varying compatibility. This leads to a rich phase diagram where we can explore how insulating phases survive when the range of the repulsive interactions is increased, and which transitions between different insulating phases can occur. The competing interactions of finite range give rise to a multitude of charge-ordered phases, which we systematically classify at the principal critical particle densities. We then investigate a hierarchy of signatures that characterize the phases and their transitions at the fundamental and phenomenological level.

Inspection of the atomic limit where the kinetic energy term vanishes allows us to systematically identify the candidate charge-ordered phases. In this limit, the phase transitions are sharp and are only driven by considerations of the interaction energy, while liquid phases are absent. The carrier mobility at a finite kinetic energy gives scope for liquid behavior that can intervene between the charge-ordered states. The consequences are investigated numerically using the infinite-system density matrix renormalization group (iDMRG) approach with a ground state represented as an infinite matrix product state (iMPS) ${ }^{40} 46$ Many of the resulting features are already visible on the phenomenological level, as we demonstrate for the experimentally accessible kinetic energy density and the bond-order parameter, which display characteristic discontinuities at many (but not all) 
of the phase transitions identified on a more fundamental level. For the latter we employ density-density correlation functions that capture long-range charge ordering, as well as the bipartite entanglement entropy, which displays characteristic scaling in critical phases.

As our main findings, we observe that depending on the compatibility of the ordered states, the liquid phase can be strongly suppressed to the extent that the transition appears to remain directly insulator-to-insulator. Furthermore, we uncover the re-emergence of simple chargeordered phases that mediate the transition between more complicated ones, and exhibit a crossover to liquid behavior at one of the phase boundaries.

We also consider the implications of disorder, which affects the charge ordering by inducing fragmentization and further suppresses liquid behavior via localization. At large disorder strength the system displays the characteristics of a universal fragmented insulating phase.

This paper is organized as follows. In Sec. III we present the model, method, and further background for this work. The charge-ordered phases of the model in the atomic limit are described in Sec. III. Section IV discusses the consequences of a finite kinetic energy, where the emergent liquid behavior is supplemented by the direct and crossover-mediated transitions between chargeordered phases described above. The disordered system is studied in Sec. V] and our conclusions are given in Sec. VI. The Appendix contains details about the classification of charge-ordered phases for principal critical particle densities in the atomic limit.

\section{MODEL, BACKGROUND AND METHODS}

We base our investigations on a model of spinless fermionic particles that move on a one-dimensional chain of size $L$ and are interacting through a finite-range repulsive potential of maximal range $p$. The disorder-free Hamiltonian of this model is given by 18

$$
H=-t \sum_{i=1}^{L}\left(c_{i}^{\dagger} c_{i+1}+\text { h.c. }\right)+\sum_{i=1}^{L} \sum_{m=1}^{p} U_{m} n_{i} n_{i+m}
$$

where $c_{i}^{\dagger}, c_{i}$ are fermionic creation and annihilation operators on site $i=1, \ldots, L, n_{i}=c_{i}^{\dagger} c_{i}$ are the corresponding particle-number operators, $t$ determines the kinetic energy, and $U_{m}$ is the interaction energy between two particles that are $m \leq p$ sites apart. All interactions are assumed to be repulsive $\left(U_{m}>0\right)$. While only the ratios $U_{m} / t$ matter for the properties of the system, we will treat these scales independently as this facilitates the discussion of the atomic limit $(t \rightarrow 0)$. The particle density is denoted as $Q=L^{-1}\left\langle\sum_{i} n_{i}\right\rangle$. Disorder can be included via a term

$$
H_{\mathrm{dis}}=\sum_{i=1}^{L} h_{i}\left(n_{i}-\frac{1}{2}\right)
$$

with uniformly distributed random potentials $h_{i} \in$ $[-W, W]$ at disorder strength $W$.

In the seminal Ref. 18, the potential energy is strictly convex $\left(U_{m+1}+U_{m-1}>2 U_{m}\right)$, which assures that there is at most one insulating phase for any given particle density $Q$ in the system. These phases can then be investigated assuming a hierarchy of well-separated energy scales $t \ll \cdots \ll U_{3} \ll U_{2} \ll U_{1}$, hence close to the atomic limit. Under these conditions the system is found to sustain a charge-ordered insulating phase at any commensurable particle density

$$
Q_{m}=1 / m, \quad m=p+1, p, \ldots, 2,
$$

while otherwise the system behaves as a Luttinger liquid.

A distinctively more non-trivial behavior can be encountered at these critical densities if the interaction potential is not convex, so that several charge-ordered states can compete at the same commensurable particle density. The convexity condition was abandoned in previous studies of the case $p=2$, where the system is also known as the $t-V-V^{\prime}$ model $19|20| 28|29| 47 \sqrt[50]{ }$ This revealed that two charge-ordered states can compete at half filling, and that the transition between these phases is mediated by a liquid phase and bond-ordered phases. In this paper, we explore this competition for the much broader range of charge orderings that occur at larger values of the interaction range $p$. In the $\left\{U_{m}\right\}$ phase diagram, this gives rise to multiple instances of charge-ordered phases separated by intervening states that mediate their transition, which are the main focus of this work.

The scene will be set by the analytical classification of charge-ordered phases in the atomic limit $t \rightarrow 0$, while the consequences of a finite kinetic energy are investigated numerically. We adopt a density-matrix renormalization group approach $\frac{40,42}{42}$ based on a tensornetwork formulation $\sqrt{51} \sqrt{53}$ where the target states are rep-

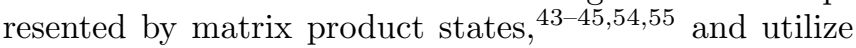
for this the Matrix Product Toolkit ${ }^{46}$ code together with our implementation of the Hamiltonian (1). This approach circumvents, e.g., the restriction to small system sizes encountered in exact diagonalization and the fermionic sign problem encountered in Quantum Monte Carlo approaches ${ }^{56}$ To investigate the ground state near the thermodynamic limit, the desired state of the system is represented as an iMPS, which accounts for an infinite number of unit cells. Note that iDMRG used in the iMPS context is different from the infinite-size algorithm of DMRG in the context of finite systems. ${ }^{43 \mid 44}$ During each step of iDMRG the filling is kept at $Q$ (the $\mathrm{U}(1)$ symmetry is preserved - for details, including a discussion of spontaneous breaking of discrete symmetries, see Ref. 44).

The iMPS unit cell size is chosen to make sure that the system is commensurable with all possible insulating phases determined in the atomic limit, whereby we avoid the frustration of any relevant charge-ordered state. Specifically, for a half-filled $(Q=1 / 2)$ system with $p=2$, the possible charge-ordered states have periods two and 
four, so that we choose a unit cell of 4 sites. For $Q=1 / 2$ but $p=4$, the phases are far more richer, which requires a unit cell of size 24 . The maximal number of saved states (bond dimension $\chi$ ) in the iDMRG procedure is 1000 .

Using these tools, we characterize the phases by a set of complementary signatures. For the most phenomenological description we consider the kinetic energy density

$$
T=\frac{1}{L}\left\langle\sum_{i=1}^{L}\left(c_{i}^{\dagger} c_{i+1}+\text { h.c. }\right)\right\rangle .
$$

This is a single-particle observable that probes the particle mobility between neighboring sites and can, in principle, be assessed in atom-optical experiments by time-offlight measurements of atoms released from the optical lattice.

The extent of bond-order is addressed by the order parameter, 20

$$
O_{\mathrm{BO}}=\frac{1}{L}\left\langle\sum_{i=1}^{L}(-1)^{i}\left(c_{i}^{\dagger} c_{i+1}+\text { h.c. }\right)\right\rangle,
$$

which constitutes a staggered version of the kinetic energy density. This parameter measures the amount of the dimerization in the system. We report its absolute value, which is invariant under the translation of the measured state.

We note that $O_{\mathrm{BO}}$ can also be finite in certain chargeordered states. This ambiguity is resolved by supplementing this quantity with additional information. The required detailed insight into the charge ordering is provided by the density-density correlation functions

$$
N_{m}=\frac{1}{L}\left\langle\sum_{i=1}^{L} n_{i} n_{i+m}\right\rangle
$$

which probe the ordering of particles that are $m$ sites apart, and allow to further discriminate charge-ordered from bond-ordered and liquid phases. To describe the long-range effects in the system, we exploit that $N_{m}$ develops an oscillating behavior in $m$. More precisely, we observe that the limit

$$
\lim _{k \rightarrow \infty} N_{m+k P}=N_{m}^{\infty}, \quad m=1, \ldots, P
$$

exists, where $P$ is the unit-cell size of the charge order. We call $N_{m}^{\infty}$ the extrapolated density-density correlator. This quantity describes the long-range charge correlations in the system.

Finally, on the most fundamental level we characterize the quantum phases and transitions by the scaling of the bipartite von Neumann entanglement entropy

$$
S=-\operatorname{tr}\left(\rho_{A} \log _{2} \rho_{A}\right)
$$

where $\rho_{A}$ is the reduced density matrix of a subchain $A$. Away from quantum-critical behavior, $S$ scales as the system's boundary (the well-known area law) $)^{57+59}$, and

\begin{tabular}{|c|c|c|c|c|c|c|c|c|}
\hline & \multirow{2}{*}{$\begin{array}{l}Q= \\
1 / 2\end{array}$} & \multicolumn{6}{|c|}{$\longleftarrow$} \\
\hline & & & $1 / 3$ & $1 / 4$ & $1 / 5$ & $1 / 6$ & $1 / 7$ & $\ldots$ \\
\hline \multirow[t]{3}{*}{$p=$} & 1 & 1 & & & & & & \\
\hline & 2 & 2 & 1 & & & & & \\
\hline & 3 & 3 & 3 & 1 & & & & \\
\hline \multirow[t]{3}{*}{$\downarrow$} & 4 & 5 & 7 & 4 & 1 & & & \\
\hline & 5 & 8 & 12 & 7 & 5 & 1 & & \\
\hline & 6 & 12 & $\geqslant 63$ & $\geqslant 23$ & 9 & 6 & 1 & \\
\hline
\end{tabular}

Table I. Number of distinct charge-ordered insulating phases in the atomic limit $t \rightarrow 0$ of the model (1), for different interaction ranges $p$ and commensurable particle densities $Q$. For details of the construction see Appendix A

therefore converges with increasing bond dimension $\chi$ in the charge-ordered and bond-ordered phases. If the system is critical, the entropy is expected to increase logarithmically with $\chi^{60[61}$, which in our investigation occurs at phase transitions and in the liquid phase. In the iDMRG algorithm, the entropy is calculated during

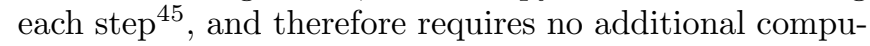
tational cost.

\section{ATOMIC LIMIT}

To prepare the investigation of quantum phase transitions between the insulating phases of different charge order, we first inspect the atomic limit of $t \rightarrow 0$ at different values of $p$ and critical densities $Q=Q_{m}$ [see Eq. (3)]. In this limit we can identify the distinct charge-ordered phases by purely combinatorial energetic considerations.

The most trivial case occurs at density $Q=Q_{p+1}$, where the fermions can be spread out evenly across the system so that they are outside of the range of their interactions. This then defines a universal ground-state with vanishing energy, which is $(p+1)$-fold degenerate.

For density $Q=Q_{p}$, the ground state can constitute any one of $p$ distinct candidate phases, which we enumerate by an index $\alpha=1, \ldots, p$. As shown in Appendix A 1 . these consist of $N /(p-\alpha+1)$ blocks of a single fermion accompanied by $(\alpha-1)$ empty sites, and $N(p-\alpha) /(p-\alpha+1)$ blocks of a single fermion accompanied by $p$ empty sites, where $N$ is the number of fermions in the considered segment. The competition between these phases is governed by their energy $E_{\alpha}=N U_{\alpha} /(p-\alpha+1)$, so that the ground-state phase $\alpha$ is selected by the condition

$$
U_{\alpha}<\frac{p-\alpha+1}{p-\beta+1} U_{\beta}, \quad \beta \neq \alpha .
$$

An important example is the case $p=2, Q=Q_{2}=$ $1 / 2$, which corresponds to the $t-V-V^{\prime}$ model at half filling studied in Refs. 19] and 20. The phase diagram then consists of two phases: one with a ground-state unit cell of $(\bullet \circ)$, where $\bullet$ is an occupied site and $\circ$ is an unoccupied site; and one with a unit cell of $(\bullet \bullet \circ)$. The two phases 
Table II. (Color online) Ground-state (GS) unit cells and their energies in the atomic limit of half-filled systems $(Q=1 / 2)$ with interaction range $p=2$ and $p=4$. In the pictorial representations of the unit cells, $\bullet$ denotes an occupied site and $\circ$ denotes an empty site. The degeneracy $f$ accounts for the translational freedom of these phases. The colors designate their position in the phase diagrams of Fig. 1

\begin{tabular}{|c|c|c|}
\hline GS unit cell & Energy density & $f$ \\
\hline \multicolumn{3}{|c|}{$p=2, Q=1 / 2$} \\
\hline$\bullet \circ$ & $U_{2} / 2$ & \\
\hline$\bullet$ & $U_{1} / 4$ & 4 \\
\hline \multicolumn{3}{|c|}{$p=4, Q=1 / 2$} \\
\hline$\bullet \circ$ & $\left(U_{2}+U_{4}\right) / 2$ & \\
\hline$\bullet \bullet \circ ০$ & $\left(U_{1}+U_{3}+2 U_{4}\right) / 4$ & \\
\hline$\bullet \bullet \bullet \circ \circ \circ$ & $\left(2 U_{1}+U_{2}+U_{4}\right) / 6$ & \\
\hline$\bullet \bullet c$ & $\left(3 U_{1}+2 U_{2}+U_{3}\right) / 8$ & 8 \\
\hline 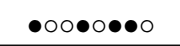 & $\left(U_{1}+2 U_{2}+3 U_{3}\right) / 8$ & \\
\hline
\end{tabular}

(a)

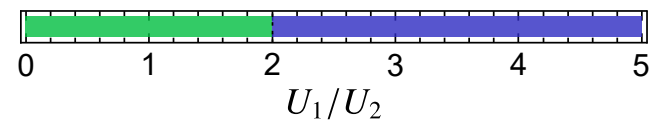

(b)

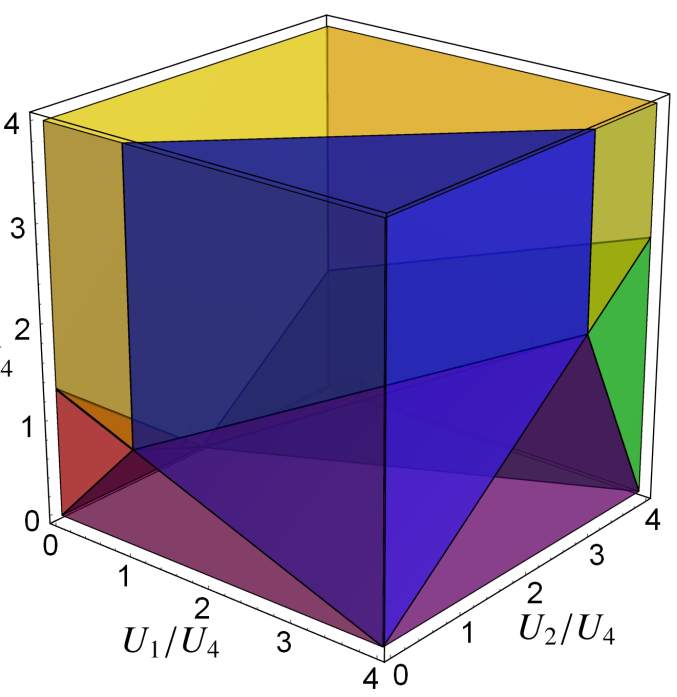

Figure 1. (Color online) Phase diagrams of charge-ordered ground states in the atomic limit of half-filled systems $(Q=$ $1 / 2$ ) with interaction range $p=2$ (a) and $p=4$ (b). In both cases we exploit the freedom to choose $U_{p}$ as the energy scale. The colors indicate the phases listed in Tab. II

have energy densities of $U_{2} / 2$ and $U_{1} / 4$, respectively, and the phase transition occurs along the $U_{1}=2 U_{2}$ line.

At higher critical densities a much wider variety of possible charge orderings emerges, whose competition can be assessed by a combinatorial analysis. Table I shows an overview of the number of insulating phases that we could determine in the atomic limit of systems with $p \leq 6$. The number of phases grows rapidly with the interaction range $p$ and depends distinctively on the density $Q$. The detailed configurations of the corresponding chargeordered states are specified in Appendix A 3. This reveals phases with highly intricate internal structure, indicating that there are no simple rules governing the ground-state properties of systems at high critical particle densities.

In the remainder of this paper we focus on the representative example of $p=4, Q=Q_{2}=1 / 2$ (hence again half filling). Table II lists the distinct charge-ordered phases for this case. Although the first four phases follow a relatively simple ordering pattern, the fifth phase displays a more intricate internal structure. The corresponding phase diagram is shown in Fig. 1, which displays the phases in the space of interaction parameters $U_{1}, U_{2}$ and $U_{3}$, while $U_{4}$ serves as the energy scale.

\section{FINITE KINETIC ENERGY}

In the atomic limit, the phase transitions between the charge-ordered phases are abrupt. This situation changes at a finite kinetic energy $t \neq 0$, where the transitions can be mediated by other phases, such as the liquid and bond-ordered phases previously encountered in the $t$ - $V$ $V^{\prime}$ model $(p=2)$. As the number of competing phases increases rapidly with larger interaction range, one could suspect that the phase space may be dominated by the transitions between these phases, while the insulating phases are only present close to the atomic limit. Thus, a large interaction range may imply the loss of insulating properties of the system. Furthermore, it is per se unclear how the distinct internal structure of the chargeordered states affects the nature of the transitions. We now explore these questions for the case $p=4$ at half filling, corresponding to the competition of ordered phases listed in Table II] and contrast this case with $p=2$. Throughout most of this section, we set $t=1$ to fix the unit of energy, and utilize the complementary signatures described in Sect. II.

For $p=2$, we find representative behavior by fixing $U_{1}=10$ while varying $U_{2}$, which allows us to verify the consistency of our results with previous studies of the $t$ $V-V^{\prime}$ model ${ }^{19 \sqrt{20}}$ For $p=4$, we find representative results by fixing $U_{1}=U_{3}=4, U_{4}=1$ and again varying $U_{2}$. According to the phase diagram in Fig. 1, this covers the region occupied by the phases $(\bullet \circ),(\bullet \bullet \bullet \circ \circ)$ and $(\bullet \bullet \circ \circ)$ in the atomic limit. These three phases are remarkably robust against the introduction of a finite kinetic energy, but to a varying degree, which leads to the unconventional transition scenarios that are the main result of this work. In contrast, the other two phases listed for $p=4$ in Tab. II occupy a smaller part of phase space and are more susceptible to suppression by a finite kinetic energy. This is illustrated at the end of this section for the phase $(\bullet \circ \bullet \bullet \bullet \bullet)$, which is quickly replaced by a bond-ordered phase.

\section{A. Phenomenological signatures}

We first consider the impact of a finite kinetic energy on the phenomenological level. This is most directly cap- 

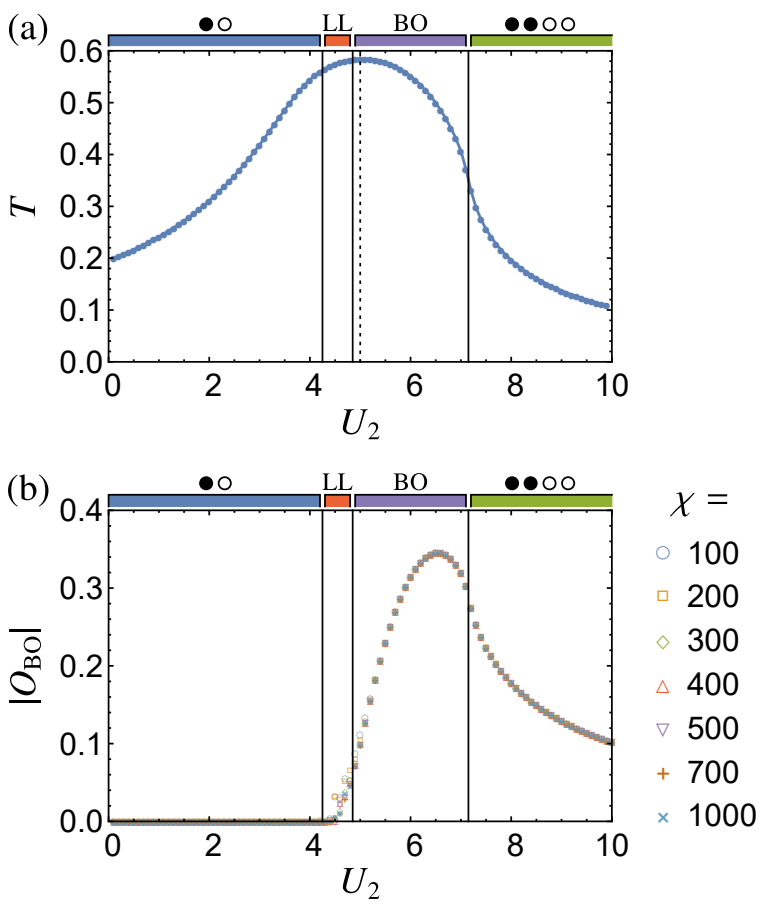

Figure 2. (Color online) Phenomenological signatures of phases and transitions in the kinetic energy density $T$ (a) and bond-order parameter $O_{\mathrm{BO}}(\mathrm{b})$ at finite kinetic energy parameter $t=1$, for interaction range $p=2$ and half filling $(Q=1 / 2)$. In both panels the next-nearest-neighbor interaction $U_{2}$ is varied while the nearest-neighbor interaction is set to $U_{1}=10$. The solid lines indicate the phase transitions in this setting, while the dotted line in (a) indicates the transition between the two charge-ordered phases in the atomic limit. The kinetic energy density only captures a single phase transition at $U_{2} \approx 7.15$, which coincides with the transition from the liquid phase into the charge-ordered phase $(\bullet \bullet \circ)$. The bond-order parameter vanishes in the thermodynamic limit of the phase $(\bullet \circ)$ and the liquid phase, but scales differently with increasing bond dimension $\chi$, thereby providing signatures of all three phase transitions.

tured by inspection of the kinetic energy density $T$ [see Eq. (4)], which is shown in panels (a) of Figs. 2 and 3 . and its staggered version, the bond-order parameter $O_{\mathrm{BO}}$ [Eq. (5)], which is shown in panels (b). We note that the range of values taken by both quantities is comparable for $p=2$ and $p=4$, which places us at a similar distance to the atomic limit. The effect of the different interaction range for both cases is immediately visible.

For $p=2$ (Fig. 2), the kinetic energy density increases as we approach the transition between the two insulating phases in the atomic limit (dotted line). The analytical behavior of $T$ resolves a single phase transition, which is signaled by a discontinuity in its first derivative (solid line). As confirmed by the bond-order parameter, this phase transition coincides with the transition from the bond-ordered phase to the phase $(\bullet \bullet \circ)$, where the latter admits a finite values of $O_{\mathrm{BO}}$ as the cuts $(|\bullet \bullet| \circ \circ \mid)$ and $(\bullet|\bullet \circ| \circ)$ are inequivalent in this phase. The liquid
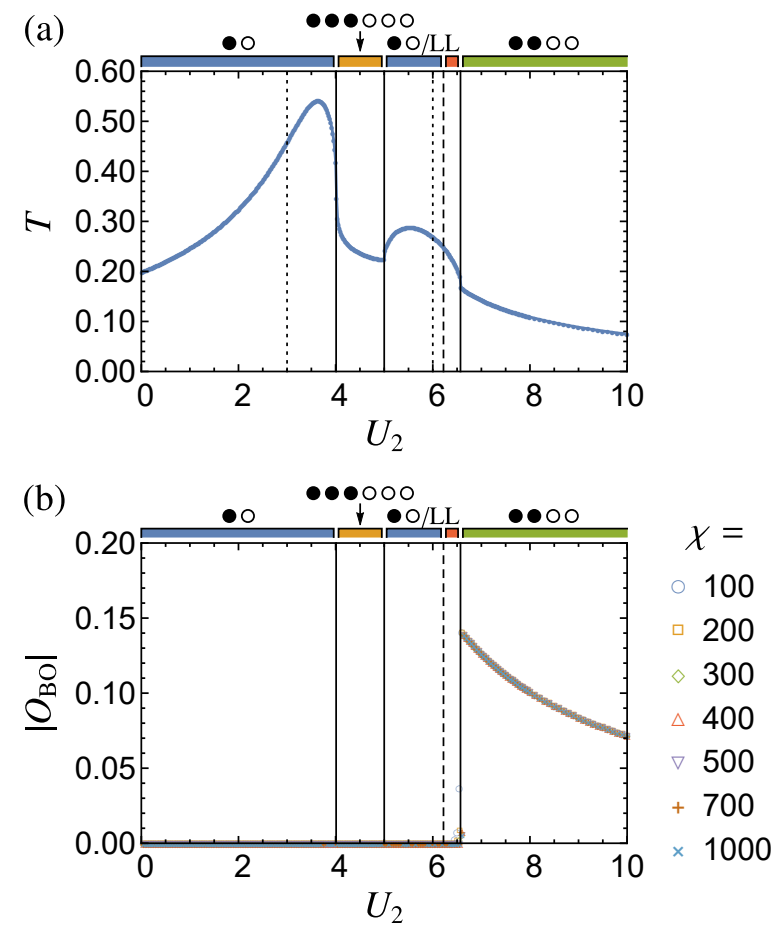

Figure 3. (Color online) kinetic energy density $T$ (a) and bond-order parameter $O_{\mathrm{BO}}$ (b) in analogy to Fig. 2, but for increased interaction range $p=4$ and interaction parameters fixed to $U_{1}=U_{3}=4$ and $U_{4}=1$. In the atomic limit, the transitions between the charge-ordered phases occur at $U_{2}=3,6$ (dotted lines). Discontinuities in the derivative of the kinetic energy density indicate three clear phase transitions at $U_{2} \approx\{4.01,5.00,6.57\}$ (solid lines). The bond-order parameter only captures a single transition into the phase $(\bullet \bullet \circ)$, which is the only encountered phase where $O_{\mathrm{BO}}$ is finite. Note that the transition between the phases $(\bullet \circ)$ and $(\bullet \bullet \circ)$ appears to be abrupt. This is verified in the subsequent figures, which also determine the indicated nature of the mediating region between the phases $(\bullet \bullet \bullet \circ \circ)$ and $(\bullet \bullet \circ)$. There, we observe a crossover from a re-emergent charge-ordered state $(\bullet \circ)$ to liquid behavior, as indicated by the dashed line.

phase is signaled by the continuing drop of $O_{\mathrm{BO}}$ with increasing bond dimension, as this order parameter has to vanish in the thermodynamic limit. The bond-order parameter also vanishes in the phase $(\bullet \circ)$, as the cuts $(|\bullet \circ|)$ and $(\bullet \mid \circ)$ are equivalent by particle-hole symmetry. The resulting sequence of phases is marked on top of the panels. The resulting picture agrees with the previous studies of the $t-V-V^{\prime}$ model in Refs. 19 and 20, where the phase diagram was determined from the bond-order parameter and the ground-state curvature. In this case, therefore, the kinetic energy density carries less detailed information than the bond-order parameter.

In contrast, for the increased interaction range $p=4$ (Fig. 33) we find distinctively more pronounced signatures of several phases already in the kinetic energy density, with three clear phase transitions indicated by the analytical behavior of $T$. As we will confirm below, these 

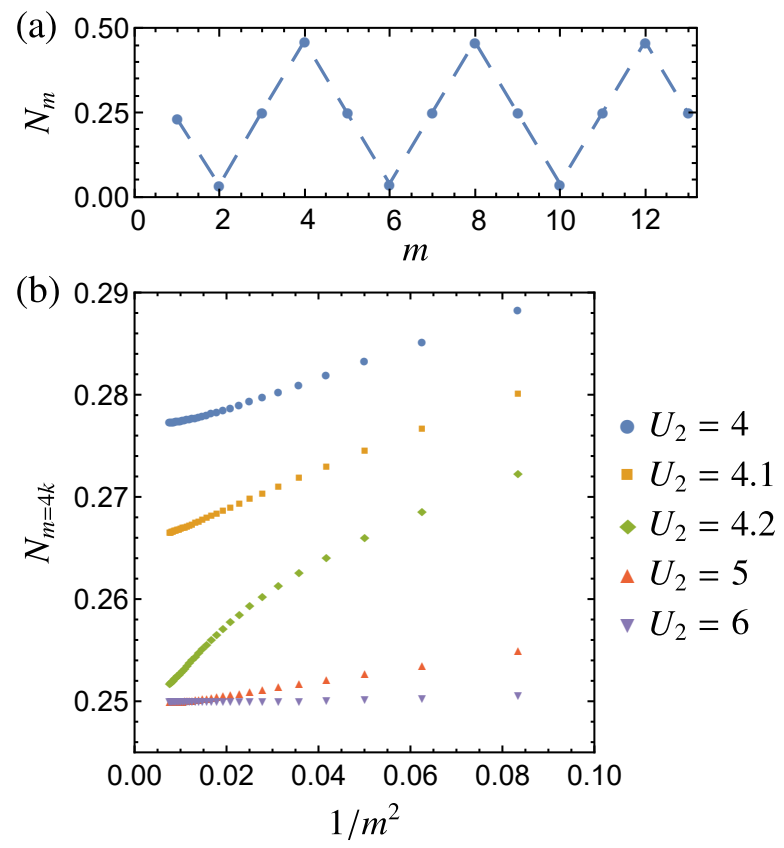

Figure 4. (Color online) (a) Range dependence of the correlation function $N_{m}$ for $p=2, Q=1 / 2, t=1$, with the interaction potentials $U_{1}=10$ and $U_{2}=8$ set to values where the system is in the charge-ordered state $(\bullet \bullet \circ)$. The function displays a clear oscillatory behavior with period 4 , reflecting that the charge-ordered character of the phase persists at finite kinetic energy. (b) Extrapolation of the correlation function $N_{4 k}$ with increasing $k$, with $U_{1}$ and $t$ as above but for various values of $U_{2}$. With increasing range $4 k$ this correlation function converges to a well-defined value $N_{4}^{\infty}$, in accordance to the extrapolated correlators stipulated in Eq. (7).

coincide with an abrupt transition between the phases $(\bullet \circ)$ and $(\bullet \bullet \bullet \circ)$ ), as if still in the atomic limit; a transition into a mediating region with partially ordered and partially liquid behavior, to which we will pay particular attention; and finally the transition into the phase $(\bullet \bullet \circ)$. The bond-order parameter now carries less insight as it is only finite in the phase $(\bullet \bullet \circ)$; in the phase $(\bullet \bullet \bullet \circ)$ it vanishes as the cuts $(|\bullet \bullet| \bullet \circ|\circ|)$ and $(\bullet|\bullet \bullet| \circ \circ \mid \circ)$ are again equivalent by particle-hole symmetry. We therefore do not detect a separate bond-ordered phase. Instead, as we will argue in the following, the mediating region contains a crossover between a re-emergent phase $(\bullet \circ)$ and a liquid phase, resulting in the sequence of phases indicated at the top of the panels.

\section{B. Correlation functions}

A more detailed characterization of the encountered phases is provided by the correlation functions $N_{m}$ defined in Eq. 6). The utility of these functions is illustrated in Fig. 4(a), where we show their $m$-dependence for a half-filled system with $p=2$ in the region where we expect the charge-ordered phase $(\bullet \bullet \circ)$. The correlation function displays an oscillating behavior in $m$, with a period $P=4$ that reflects the size of the charge-ordered unit cell. As shown in Fig. 4(b), the limiting quantities $N_{m}^{\infty}$ given in Eq. (7) are indeed well defined.

With these features, the correlation functions give direct insight into the charge-ordered character of the phases. In the atomic limit, the extrapolated correlators take values $N_{m}^{\infty} \in\{i / P\}$ if the system is in the insulating phase, where $i$ is an integer. The chargeordered phase with a unit cell $(\bullet \circ)$ displays two alternating values 0 and $1 / 2$, the phase $(\bullet \bullet \circ)$ admits three possible values alternating as $(1 / 4,0,1 / 4,1 / 2)$, and the phase $(\bullet \bullet \bullet \circ \circ)$ admits four possible values alternating as $(1 / 3,1 / 6,0,1 / 6,1 / 3,1 / 2)$. For $t>0$, the precise values of $N_{m}^{\infty}$ in a charge-ordered phase are expected to deviate from the atomic limit, but their periodicity and the ordering of the encountered values should be preserved. In the liquid phase, we expect the long-range correlations to become trivial. The average correlation function between any two positions in the system should therefore acquire the value $\left\langle n_{i} n_{i+m}\right\rangle / L^{2} \approx\left\langle n_{i}\right\rangle\left\langle n_{i+m}\right\rangle / L^{2}=Q^{2}=1 / 4$, where we specified the case of half filling. While these features clearly separate all charge-ordered states, the same value $1 / 4$ is also obtained in the bond-ordered phase, which we detected above with the bond-order parameter.

Fig. 5 shows the extrapolated correlators $N_{m}^{\infty}$ for both investigated systems under the same conditions as in Figs. 2 and 3 All charge-ordered phases can be clearly identified using the expected periodicity from the atomic limit. We notice that there are regions where all the correlators reach the value $1 / 4$, indicating the absence of charge order. The discontinuities in the derivative of $N_{m}^{\infty}$ coincide with the phase transitions detected by $T$ and $O_{\mathrm{BO}}$.

For $p=4$, these results confirm that the transition between the phases $(\bullet \circ)$ and $(\bullet \bullet \bullet \circ \circ)$ appears to be sharp, with an undetectable intervening liquid phase. Significantly, the mediating region between the phases $(\bullet \bullet \bullet \bullet \circ)$ and $(\bullet \bullet \circ)$ indeed exhibits the signatures of the phase $(\bullet)$. Furthermore, as shown in detail in Fig. 6, the values of $N_{m}^{\infty}$ get progressively closer to $1 / 4$, indicating a possible crossover into the liquid state, as suggested by the label $(\bullet \circ) / \mathrm{LL}$.

\section{Entanglement entropy}

To further resolve the details of the mediating transition region we turn to the entanglement entropy, which is presented in Fig. 7. In general, in ordered states the entropy can take multiple values depending on the position of the cut that bipartites the system. In the case of $p=2$ (panel a), the mirror symmetry of the insulating phase $(\bullet \circ)$ implies that $S$ remains single-valued, while the insulating phase $(\bullet \bullet \circ)$ has two possible values, where we account for particle-hole symmetry and mirror symmetry. In the bond-ordered phase, the ground state is char- 

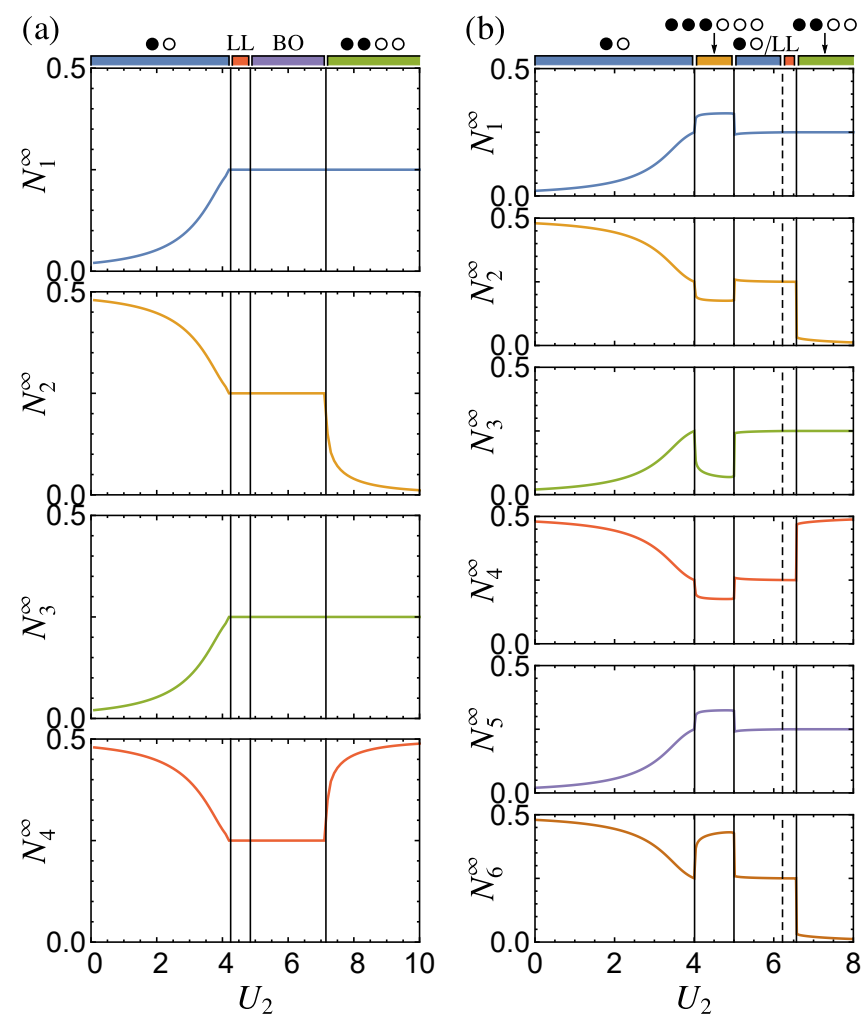

Figure 5. (Color online) Extrapolated correlators $N_{m}^{\infty}$ for (a) $p=2$ and (b) $p=4$, with system parameters specified as in Figs. 2 and 3 The phase transitions indicated by the solid lines coincide with points where the derivative of $N_{m}^{\infty}$ is discontinuous. The oscillatory behavior of the correlators with the range $m$ agrees with the stipulated charge orders. For $p=4$, the transition between the phases $(\bullet \circ)$ and $(\bullet \bullet \bullet \circ \circ)$ remains abrupt. The behavior in the mediating transition region between the phases $(\bullet \bullet \bullet \circ \circ)$ and $(\bullet \bullet \circ)$ is examined more closely in Fig. 6 .

acterized by an alternating local structure, and therefore $S$ again has two possible values. Finally, in the liquid phase the entropy is single-valued, but does not converge with increasing bond dimension. Therefore, the entropy also allows to discriminate the bond-ordered and liquid phases. The transitions between the different phases are clearly visible in these numerical results, and agree with the signatures described above.

Figure 7(b) shows the entanglement entropy for the half-filled system with $p=4$. The entropy can again be multivalued, where the charge-ordered phases $(\bullet \circ)$ and $(\bullet \bullet \circ)$ and the liquid phase behave in analogy to the case $p=2$. In the phase $(\bullet \bullet \bullet \circ \circ)$ the entropy can have two values, where we again account for mirror symmetry and particle-hole symmetry. As anticipated above, the entropy does not detect any indications of a liquid phase between the phases $(\bullet \circ)$ and $(\bullet \bullet \bullet \circ)$. We also do not find any indications of the bond-ordered phase, which may be attributed to the modified energetic conditions from the additional interaction terms. Most importantly, the results confirm that the transition between the phases

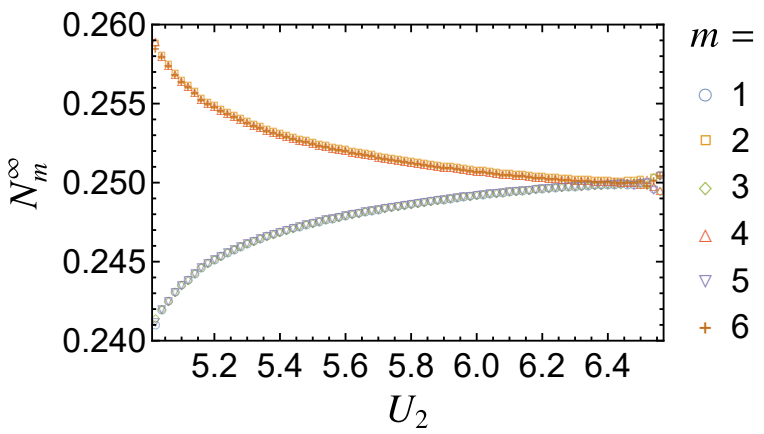

Figure 6. (Color online) Close-up of the extrapolated correlators $N_{m}^{\infty}$ from Fig. 5 for the mediating transition region in the system with $p=4$. The correlations gradually approach the value $1 / 4$, compatible with a gradual crossover from charge order $(\bullet \circ)$ to liquid behavior, where furthermore the results converge only slowly.

$(\bullet \bullet \bullet \circ)$ ) and $(\bullet \bullet \circ)$ is mediated by an ordered state that shares all signatures with the phase $(\bullet \circ)$. As the ordering in this state approaches the liquid behavior according to the correlations in Fig. 6, the system develops the features of a liquid state, where the entropy continues to increase with increasing bond dimension. Within the numerically accessible bond dimensions this takes the form of a transition as indicated above the panel, but it is also plausible that this behavior indicates a crossover with a rapidly increasing convergence threshold in the bond dimension.

\section{Extended phase diagram}

According to the picture developed above, the phase $(\bullet \circ)$ is present twice in the phase diagram explored thus far, where it surrounds the phase $(\bullet \bullet \bullet \circ \circ)$. As a phenomenological explanation for this re-emergent behavior once could suggest that the phase $(\bullet \circ)$ mediates the transition between the phases $(\bullet \bullet \circ)$ and $(\bullet \bullet \bullet \circ \circ)$ as its simpler structure reflects the required chargereconfigurations between the latter two phases. Further insight into the mechanism behind this reemergence is obtained by sampling the parameter space more widely. For this we keep the potential energies $U_{1}=U_{3}=4, U_{4}=1$ fixed as before and continue to vary $U_{2}$, but also consider values of $t \neq 1$ that supplement the results presented earlier in this section.

We recall that for $t=0$, the system is in the atomic limit (see Sec. III), where direct transitions between the charge-ordered phases occur at $U_{2}=3$ and $U_{2}=6$. For $t \rightarrow \infty$, we expect the phase diagram to display the liquid phase, as the Hamiltonian is then dominated by the kinetic term. While we cannot map out the full phase diagram with a high degree of precision, reasonable estimates of the phases and their transitions are obtained by limiting the bond dimension $\chi$ to 200 and 400 for representative values of $t$. This leads to the extended phase 

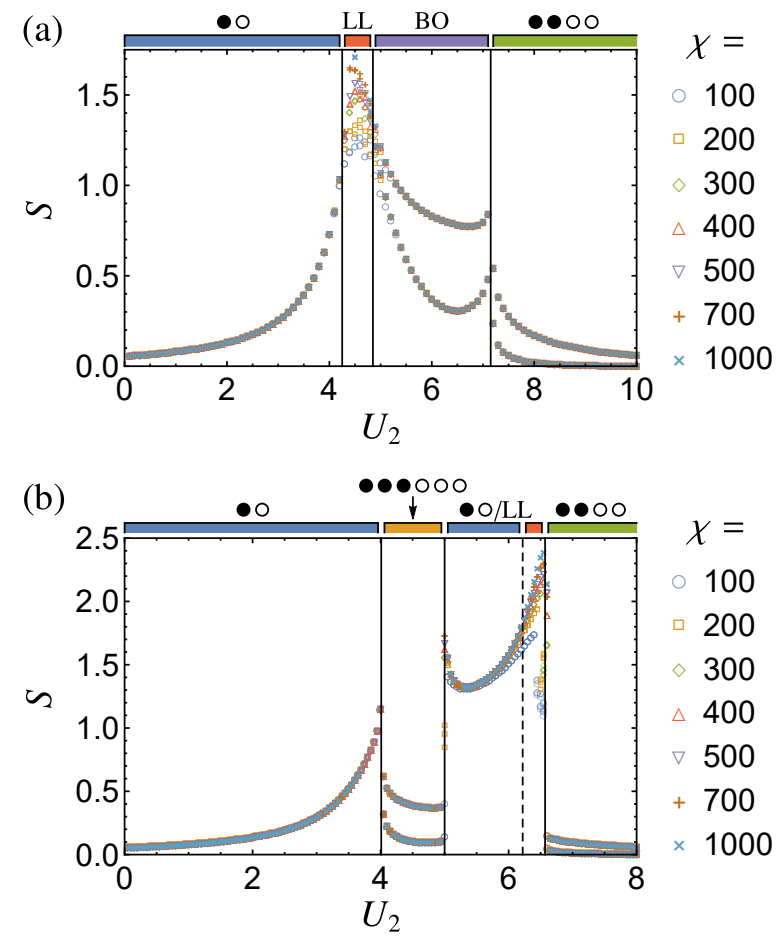

Figure 7. (Color online) Bipartite entanglement entropy for the systems with $p=2$ (a) and $p=4$ (b), with parameters as specified in Figs. 2 and 3 For $p=4$, no critical behavior is detected around the transition between the phases $(\bullet \circ)$ and $(\bullet \bullet \bullet \circ)$. However, in the transition region between the phases $(\bullet \bullet \bullet \circ)$ and $(\bullet \bullet \circ)$ the gradual suppression of charge correlations (Fig. 6 coincides with an onset of finitesize scaling with increasing bond-dimension, as expected from a crossover into a liquid phase.

diagram proposed in Fig. 8. Here, most transitions are captured accurately with good agreement between the signatures from the extrapolated correlators and the entropy. The determination of the transition between the phase $(\bullet \circ)$ and the liquid phase requires very high bond dimensions, so that its approximate position at the accessible bond dimensions is marked by a dashed line.

As can be seen from the diagram, the phase $(\bullet \bullet \bullet \circ \circ)$ only exists up to moderate values of the kinetic energy parameter $t$, while the phase $(\bullet \circ)$ is distinctively more robust, wraps around the other phase, and indeed reemerges in the mediating transition region along the $t=1$ line. At first sight, one would expect that this re-emergent behavior cannot extend all the way to the atomic limit, as the fully charge-ordered state $(\bullet \circ)$ acquires a larger energy than the other charge-ordered states. However, a consistent scenario would see the mediating state to gradually lose its clear charge order and cross over into the liquid phase, in analogy to the behavior witnessed in the correlations of Fig. [6 and the entropy in Fig. 7. This assertion is difficult to verify, as the mediating phase becomes confined to a very small part of phase space as one approaches the atomic limit. This complication does not apply to the region $t \approx 1$, where

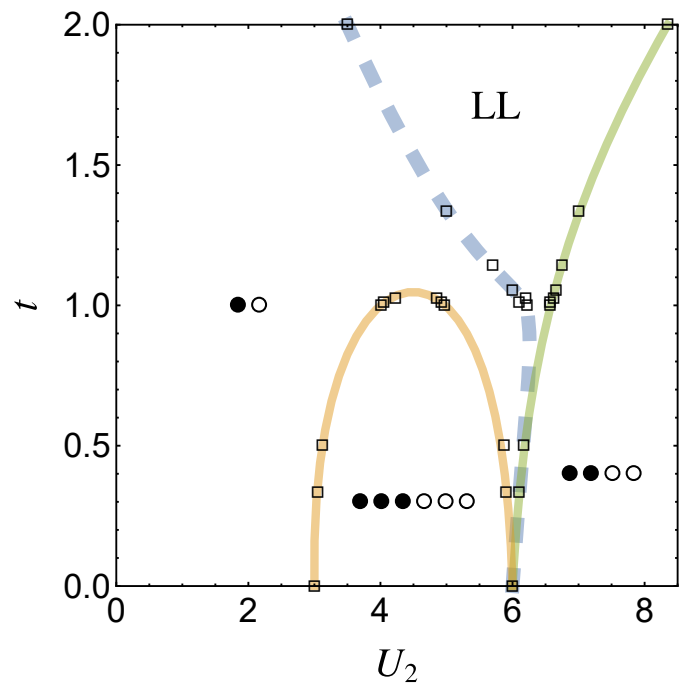

Figure 8. (Color online) Proposed phase diagram for the model from Eq. (1) with interaction range $p=4$, with interaction parameters $U_{1}=U_{3}=4, U_{4}=1$ while $U_{2}$ and the kinetic energy parameter $t$ are varied. The phase transitions are determined from the extrapolated correlators and the entanglement entropy, in analogy to Figs. 5 and 7, which correspond to the case $t=1$. The dashed line indicates the detected onset of finite-size scaling with the bond dimension in the crossover from the phase $(\bullet \circ)$ to the liquid phase.

the $(\bullet \circ)$ phase clearly wraps around the $(\bullet \bullet \bullet \circ \circ)$ phase, resulting in its re-emergent behavior.

\section{E. Fragile phases and bond order}

According to Table II, for $p=4$ the considerations above cover three of the five possible charge-ordered phases identified in the atomic limit. As the phase $(\bullet \bullet \bullet \bullet \circ \circ)$ is confined to a small part of parameter space already in the atomic limit (see Fig. 1), we here illustrate the susceptibility to a finite kinetic energy for the phase $(\bullet \circ \bullet \bullet \bullet \circ)$, which displays the most complex charge order. To explore this phase we set $U_{1}=4, U_{3}=U_{4}=1$ and again vary $U_{2}$. We then find that the phase is absent at $t=1$, but can be detected if we significantly reduce the kinetic energy parameter to $t=0.1$. The corresponding results are shown in Fig. 9. We find clear signatures of three charge-ordered phases, which occur in the sequence $(\bullet \circ),(\bullet \circ \bullet \bullet \bullet \bullet),(\bullet \bullet \circ)$ in agreement with the atomic limit. However, the support of the phase $(\bullet \circ \bullet \bullet \bullet \bullet)$ is already much reduced. This occurs in favor of two surrounding regions that both support a purely bond-ordered phase with no residual charge order, and thereby share the same characteristics as the bond-ordered phase encountered for $p=2$. Note that the phase $(\bullet \circ \bullet \bullet \bullet \bullet)$ also admits a finite bond-order parameter, as does again the phase $(\bullet \bullet \circ)$ ) already discussed above. These results not only demonstrate the suscepti- 

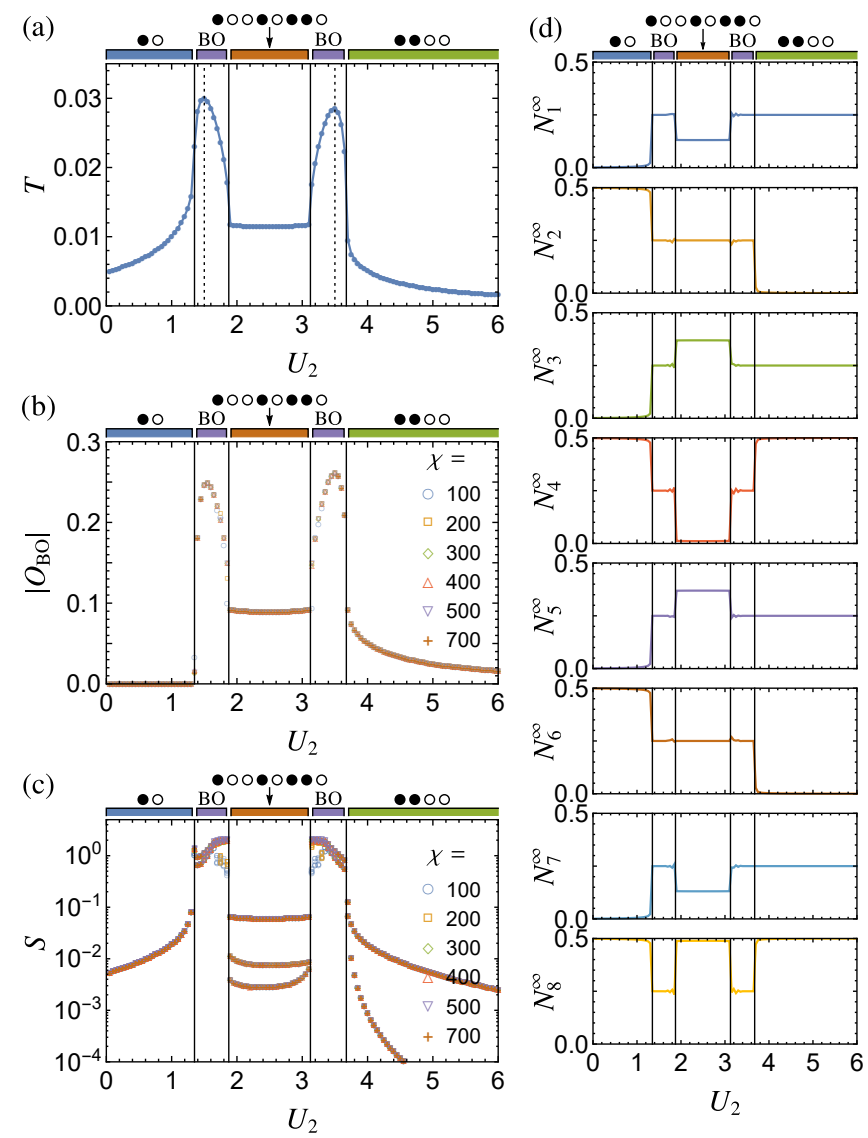

Figure 9. (Color online) Effect of a small kinetic energy parameter $t=0.1$ on the phase $(\bullet \circ \bullet \bullet \bullet \bullet \circ)$ in the half-filled system with $p=4$, as captured by the kinetic energy density $T$ (a), the bond-order parameter $O_{\mathrm{BO}}(\mathrm{b})$, the bipartite entanglement entropy $S$ (c), and the extrapolated correlators $N_{m}^{\infty}$ (d). In contrast to the case described in Fig. 3, we now set $U_{1}=4$ and $U_{3}=U_{4}=1$, while varying $U_{2}$ as before. Already at the chosen small kinetic energy parameter, the phase $(\bullet \circ \bullet \bullet \bullet \bullet)$ is driven out by a bond-ordered phase, which intervenes in the transition to the other charge-ordered states covered in this parameter range.

bility of the phase $(\bullet \circ \bullet \bullet \bullet \bullet \circ)$ to suppression by a finite kinetic energy, but also show that bond-ordered phases can still occur at this increased interaction range.

\section{EFFECTS OF DISORDER}

We now determine the effects of disorder, which is introduced into the Hamiltonian according to Eq. 22). To study these effects numerically within the adopted framework we choose disorder configurations that remain compatible with the previously encountered charge-ordered states, so that these do not experience any artificial frustration. This requires a disordered unit cell of size $L$ that is commensurable with all the possible insulating phases present in the atomic limit, hence a multiple of 4 in the half-filled system with $p=2$ and a multiple of 24 in the
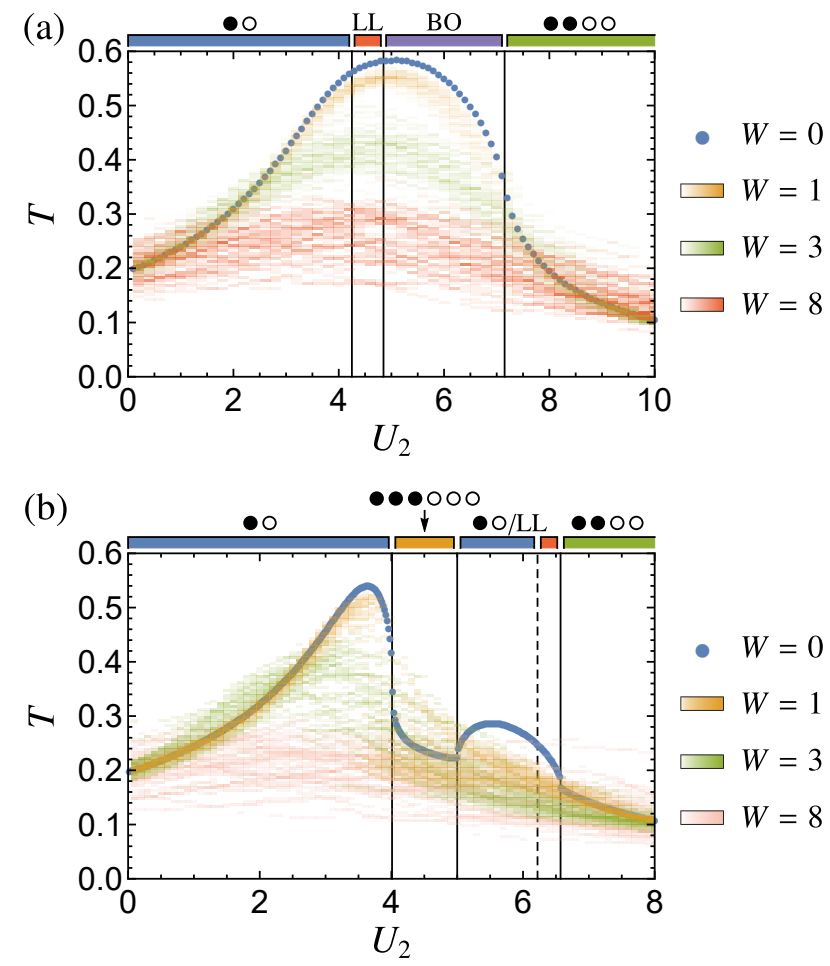

Figure 10. (Color online) Effect of disorder on the kinetic energy density for $p=2$ (a) and $p=4$ (b). The disorder-free case corresponds to Figs. 2 and 3 and is included for guidance. For each finite value of the disorder, the data represent a density plot accumulated over 100 disorder realizations. The size of the disordered unit cell is $L=20(p=2)$ and $L=24$ $(p=4)$.

half-filled system with $p=4$. By inspecting the variations of our results for $T, O_{\mathrm{BO}}$ and $S$ with $L$ for moderate to strong values of the disorder, we have found it sufficient to set $L=20$ for $p=2$ and keep $L=24$ for $p=4$, which has the added benefit of retaining nontrivial extrapolated correlators $N_{m}^{\infty}$ as discussed below. The limit of very weak disorder would require an ever-increasing disordered unit cell that keeps up with the increasing localization length, which is beyond the practical scope of the adopted iDMRG/iMPS approach.

In the atomic limit $t=0$, disorder encourages the fragmentization of charge-ordered states as the energy expense of a charge configuration can be overcompensated by the energetic gain from the on-site potential. Furthermore, previously degenerate configurations such as $(\bullet \bullet \circ),(\circ \bullet \bullet),(\circ \circ \bullet \bullet)$ and $(\bullet \circ \bullet)$ now acquire different energies. These reconfigurations have a direct effect on the long-range correlations, which can be expected to persist also at finite kinetic energy $t \neq 0$. In this general case, we would expect the disorder also to localize the liquid phase, so that the phase space regions with critical behavior should be suppressed.

On the phenomenological level these anticipated tendencies are again well captured by the kinetic energy density $T$, as shown by the disorder-averaged density plots 

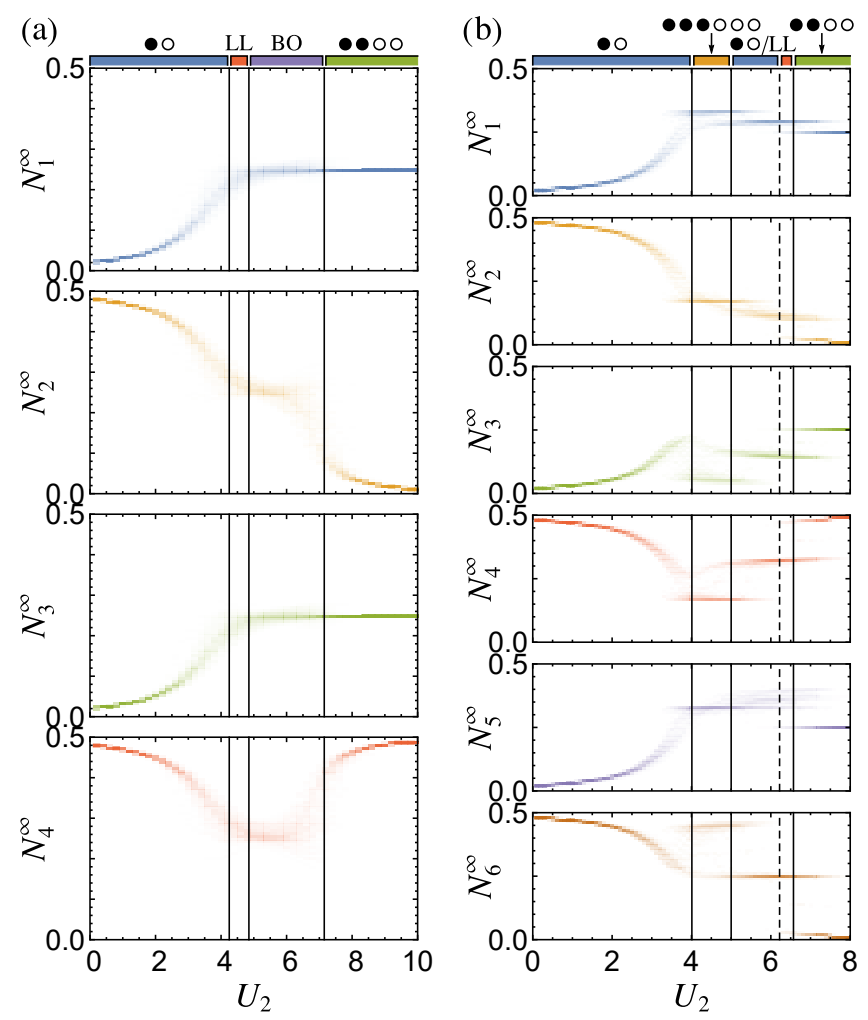

Figure 11. (Color online) Disorder-averaged density plots of the extrapolated correlators $N_{m}^{\infty}$ for the disordered systems specified in Fig. 10, evaluated at disorder strength $W=1$. These correlators remain nontrivial because of the finite size of the disordered unit cell.

in Fig. 10. For $p=2$ (panel a) the density $T$ drops significantly for increasing disorder strength $W$, in particular in the transition region between the charge-ordered phases. At the same time the spread of values of $T$ increases, and all the features present on the $W=0$ plot become progressively washed out so that at $W=8$ the kinetic energy becomes essentially independent of $U_{2}$. A similar trend is present for $p=4$ (panel b), where the two prominent peaks present for $W=0$ get washed out as one increases the disorder strength. These results are consistent with the formation of a universal fragmented state at large disorder strength.

Insight into the gradual formation of such a state is given by the correlation functions. Figures 11, 12 and 13 show disorder-averaged density plots of the extrapolated correlators $N_{m}^{\infty}$ for disordered systems with $W=1$, $W=3$ and $W=8$, respectively. Note that these correlators are expected to be trivial (equaling 1/4) at any finite disorder strength in the thermodynamic limit $L \rightarrow \infty$, but here retain a nontrivial structure as $L$ is finite. For a small disorder strength ( $W=1$, Fig. 11), the correlators for $p=2$ behave very similarly to the non-disordered case. On the other hand, for $p=4$ the same disorder strength already has a distinct effect on the mediating transition region between the phases $(\bullet \bullet \bullet \circ \circ)$ and
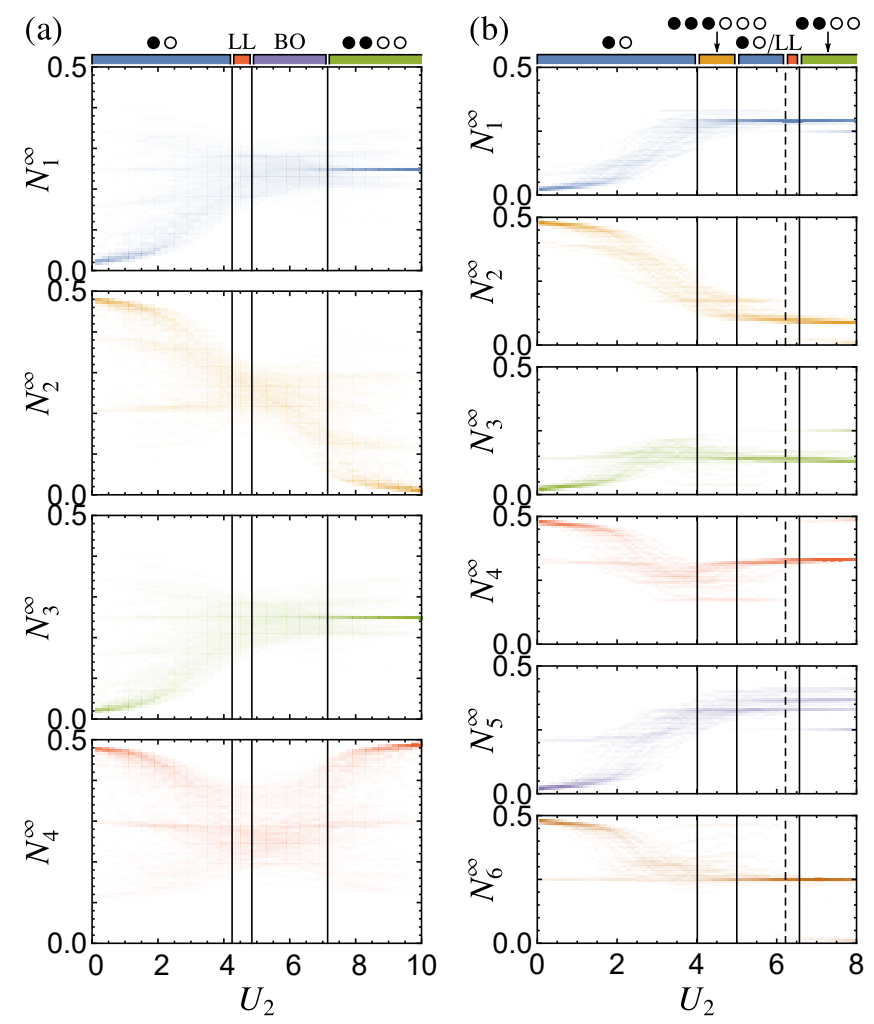

Figure 12. (Color online) Disorder-averaged density plots of the extrapolated correlators $N_{m}^{\infty}$ as in Fig. 11] but for disorder strength $W=3$.

$(\bullet \bullet \circ)$, where the re-emergent phase $(\bullet \circ)$ and the liquid phase are quickly replaced in favor of a disordered insulating phase with a nonuniform charge structure. As we increase the disorder $(W=3$, Fig. 12), the correlators $N_{m}^{\infty}$ develop distinct ridges close to rational values $i / L$, with $i=0, \ldots, L / 2$ (emphasizing again the role of the finite disordered unit cell). These ridges are most prominent in the ranges formerly occupied by the chargeordered states, where this behavior is consistent with their fragmentization. In the former transition regions the correlators cover a broad and continuous range of values. For strong disorder ( $W=8$, Fig. 13), however, the rational ridges spread out over the whole parameter range, which is consistent with the emergence of a universal fragmented insulating state. Interestingly, this state still appears to carry some characteristic ordering features. For example, in the system with $p=2$, the correlators $N_{2}^{\infty}$ and $N_{4}^{\infty}$ prefer rational values $L / i$ with even $i$.

Finally, as shown in Fig. 14 the disorder also has a significant effect on the entanglement entropy. In particular, the entropy develops strong sample-to-sample fluctuations already for small disorder strength, and its value in the previously liquid phase is already noticeably reduced. For large disorder strength the entanglement entropy becomes very small across the whole parameter range. 

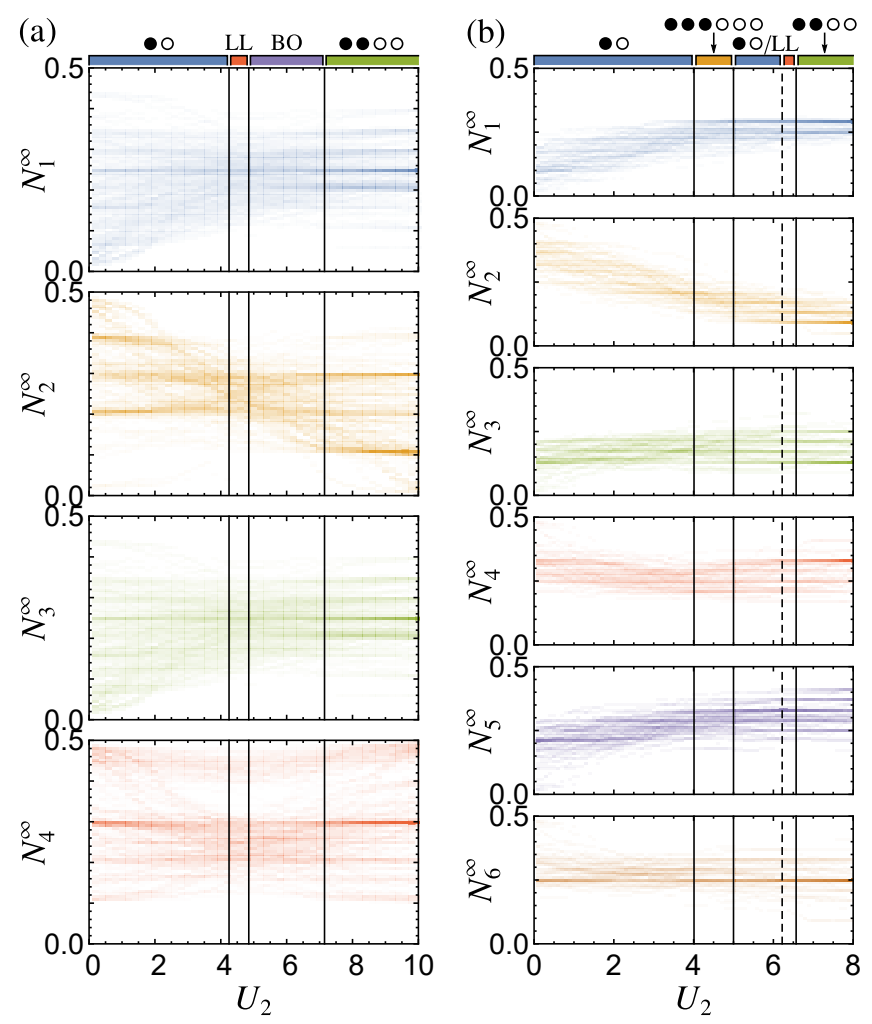

Figure 13. (Color online) Disorder-averaged density plots of the extrapolated correlators $N_{m}^{\infty}$ as in Figs. 11 and 12 but for disorder strength $W=8$.

\section{CONCLUSIONS}

In summary, we have investigated the interplay of charge-ordered fermionic insulating phases that arise from the competition of finite-range interactions in one dimension, based on the fermionic lattice model in Eq. (1) and a range of phenomenological and fundamental quantities described in Sec. II In the atomic limit of a vanishing kinetic energy term, we observe a proliferation of competing phases, which for large interaction range can display remarkably rich internal ordering (see Sec. III and the Appendix). For a finite kinetic energy (Sec. IV), significant differences are found already for moderate ranges $p$ of the interactions, as we explored by comparing the cases $p=2$ (for which we recover the known phenomenology of phase transitions mediated by liquid and bondordered phases) and $p=4$. While in the latter case some complex charge-ordered phases are quickly suppressed by a finite kinetic energy (see e.g. Fig. 9), we observe that the increased variety of competing phases with increasing interaction range does not imply an essential loss of insulating properties of the system; see the corresponding panels and phase diagrams in Figs. 3, 5, and 7. Instead, we observe, as our two main results, the survival of apparently direct transitions between two charge-ordered phases mimicking the atomic limit, as well as the appearance of mediating phases that display re-emergent
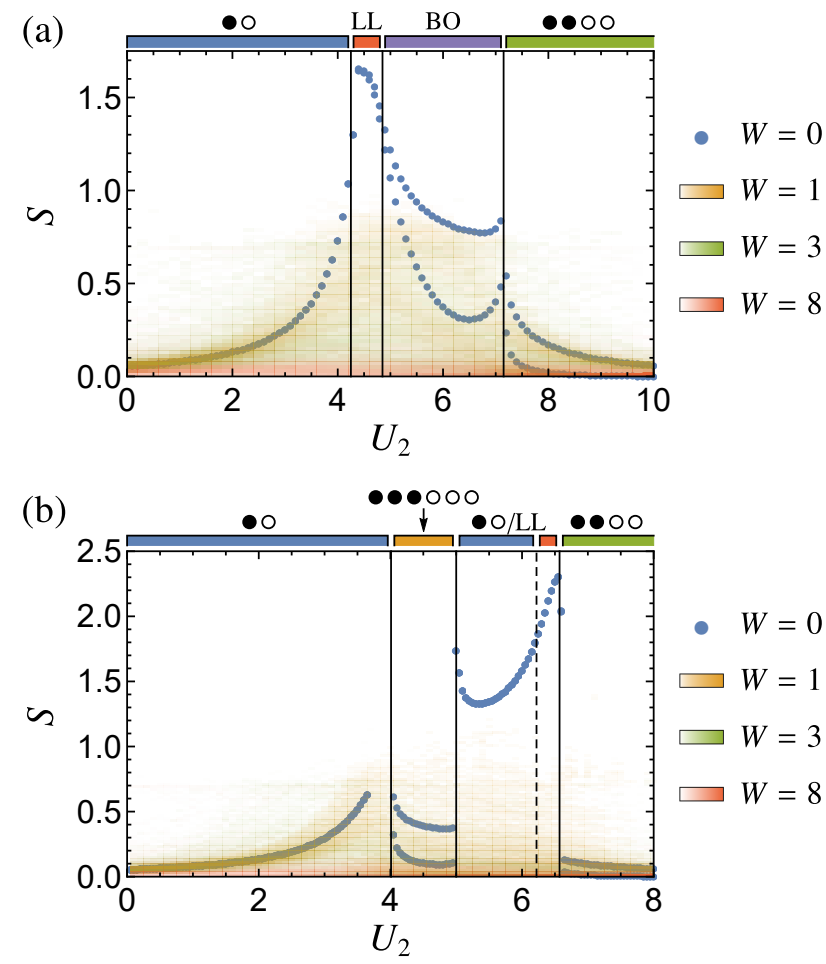

Figure 14. (Color online) Disorder-averaged density plots of the entanglement entropy $S$ for the disordered systems specified in Fig. 10 .

simple charge order and exhibit a crossover to liquid behavior at one of the phase boundaries (see in particular Fig. 6). These two transition scenarios supplement the liquid and bond-ordered phases encountered in previous studies with a small interaction range, leading to a rich variety of phases, transitions and crossovers in the system (see Fig. 8). Disorder (explored in Sec. V) has the expected effect of gradual fragmentization and localization of the insulating and liquid phases, which is particularly visible in the density-density correlation functions of a finite disordered unit cell (Figs. 11,13).

The results in this work are based on an analytical classification of charged-ordered states in the atomic limit and extensive numerical investigations at a moderate finite kinetic energy, both applied to a canonical model of interacting spinless fermions on a discrete onedimensional chain. Complementary approaches could provide useful insights into the exact nature of the observed transitions and crossovers. Numerically, this could be achieved by exploring the limit of a very large or small kinetic energy, in which the approach adopted in this work scales less favorably, or by the investigation of alternative models, such a spin chains or spinful fermions. Further analytical progress could be made perturbatively close to the atomic or free limit, or phenomenologically by field-theoretical approaches of effective, possibly continuous counterparts of the studied system. In general, our work should motivate efforts to identify and clas- 
sify the possible transition scenarios in systems where the kinetic energy competes with several interactions of different range. This competition should also persist for excited states, including for disordered systems that may display many-body localization. $\frac{62[63}{6}$ These endeavors are left for future considerations.

\section{ACKNOWLEDGMENTS}

We gratefully acknowledge useful discussions with Neil Drummond, Jens Bardarson, and Fabian Heidrich-Meisner. This research was funded by EPSRC via Grant No. EP/P010180/1. Computer time was provided by Lancaster University's High-End Computing facility. All relevant data present in this publication can be accessed at http://dx.doi.org/10.17635/ lancaster/researchdata/234.

\section{Appendix A: Identification of charge-ordered insulating phases in the atomic limit}

In this Appendix we provide classifications of chargeordered phases at principal critical densities $Q=Q_{m}=$ $1 / m$ for interaction ranges $p \leq 6$. We start with the instructive case of $Q=Q_{p}=1 / \bar{p}$, where the classification can be carried out for all $p$.

\section{Critical density $Q=1 / p$}

Assume for the moment that $U_{p} \ll U_{m}$ so that the preferable distance between two fermions is $p$. We then say that $U_{p}$ orders the fermions in the ground state. For example, a charge sequence $\bullet \underbrace{\circ 0 \cdots \circ}_{p-1} \bullet \underbrace{00 \cdots \circ}_{p-1} \bullet$ has a lower energy than the sequence $\bullet \underbrace{\circ 00 \cdots \circ}_{p} \bullet \underbrace{0 \cdots \circ}_{p-2} \bullet$. The ground state has the simple form

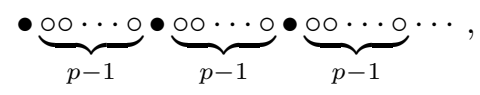

and its energy is $E_{1}=(L / p) U_{p}=N U_{p}$, where $L$ is the considered system size and $N=L / p$ the number of particles.

Next let us inspect how a low value of $U_{p-1}$ can order the fermions. This cannot be based on a repeating sequence of segments $\bullet \underbrace{00 \cdots o}_{p-2}$, as this would not result in the correct density $1 / p$. However, by addition of segments $\bullet \underbrace{00 \cdots 0}_{p}$ we can tailor the density without changing the energy of the system. A representative corresponding ground-state configuration is

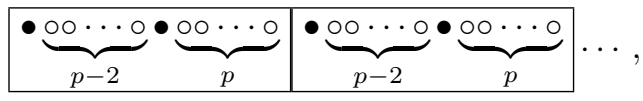

which gives us the correct density $Q=1 / p$, and results in the energy $E_{2}=(L / 2 p) U_{p-1}=(N / 2) U_{p-1}$. Note that this ground state is highly degenerate - the sections of $p$ and $p-2$ unoccupied sites can be freely arranged along the system; e.g., all sections with $p-2$ unoccupied sites could be placed besides each other without changing the energy of the system.

If one follows this prescription for the general case of ordering driven by $U_{p-n}$, one obtains ground states of the representative structure

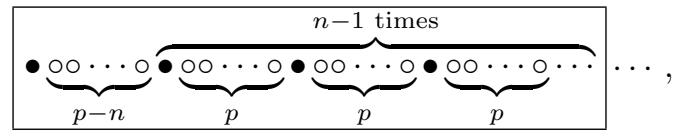

which have an energy

$$
\begin{aligned}
E_{n} & =\frac{L}{1+p-n+(n-1)(p+1)} U_{p+1-n} \\
& =\frac{L}{n p} U_{p-n+1}=\frac{N}{n} U_{p-n+1} .
\end{aligned}
$$

Again, these ground states are highly degenerate.

We can now determine the conditions in which an arbitrary phase (designated by step $n$ ) will dominate the charge ordering. This requires

$$
\underset{k \neq n}{\forall} E_{n}<E_{k} \Rightarrow \underset{k \neq n}{\forall} U_{p-n+1}<\frac{n}{k} U_{p-k+1} .
$$

Renaming $\alpha=p-n+1$ and $\beta=p-k+1$, we arrive at the condition

$$
\underset{\beta \neq \alpha}{\forall} U_{\alpha}<\frac{p-\alpha+1}{p-\beta+1} U_{\beta},
$$

which in the main text is expressed as Eq. (9.

If this condition is fulfilled then the phase with energy $E_{\alpha}=[N /(p-\alpha+1)] U_{\alpha}$ is dominant and the ground state consists of $N /(p-\alpha+1)$ segments $\underbrace{\circ \circ \cdots \circ}_{\alpha-1}$ and $N(p-\alpha) /(p-\alpha+1)$ segments $\bullet \underbrace{\circ 0 \cdots \circ}_{p}$. The groundstate degeneracy is given by

$$
f=\left\{\begin{array}{cc}
\left(\begin{array}{c}
N \\
N /(p-\alpha+1)
\end{array}\right) \cdot p & \text { if } 2 \alpha>p \\
N \frac{p-\alpha}{p-\alpha+1} \\
N /(p-\alpha+1)
\end{array}\right) \cdot \frac{p(p-\alpha+1)}{p-\alpha} \text { otherwise. }
$$

For $2 \alpha \leqslant p$, the degeneracy count reflects the requirement to exclude cases where blocks of structure $\bullet \underbrace{\circ 0 \cdots 0}_{\alpha-1}$ are adjacent, which then increases their energy by $U_{2 \alpha}$.

\section{General properties at higher critical densities}

To construct the charge-ordered phases at larger critical densities $Q_{m}=1 / m$ with $m<p$, we rely on the following two general properties: 
Property 1 In any atomic charge configuration of density $Q$, there is at least one sequence of $1 / Q-1$ or more unoccupied sites.

Proof. When the particles are evenly spread out over the system they are $1 / Q$ sites apart, i.e., separated by $1 / Q-1$ unoccupied sites, corresponding to the configuration

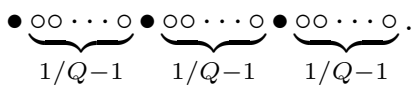

Any attempt to reduce one of these spacings further necessarily increases another spacing.

Property 2 For any atomic ground state of the system, the largest sequence of unoccupied states cannot exceed $p$ sites.

Proof. Assume that there exists a ground state unit cell with a sequence of $(p+1)$ unoccupied sites, which we place to the very right in the cell by exploiting translational invariance. A periodic arrangement of these unit cells then takes the form

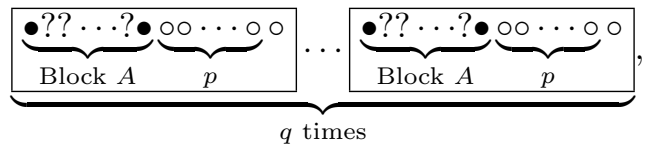

and has an energy density $E_{A} /(N / Q)$ where $E_{A}$ is the energy of the denoted block $A$. We can now move the right-most unoccupied sites in each cell to the very end of this chain without changing the energy density, resulting in the rearranged configuration

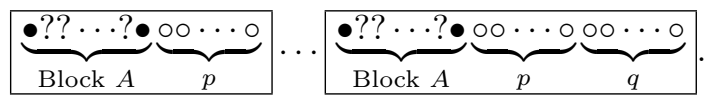

As guaranteed by the finite density $Q>1 / p+1$, block $A$ contains at least one particle that makes a finite contribution $E_{\Delta}$ to the energy of this state. We can now take this particle from block $A$ and place it into the terminating segment of the chain,

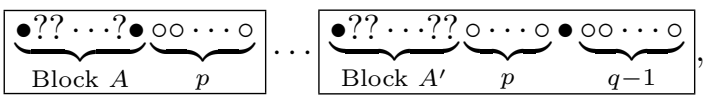

where block $A^{\prime}$ is block $A$ with the particle replaced by a hole. Block $A^{\prime}$ has energy $E_{A}-E_{\Delta}$, while the displaced particle no longer contributes to the energy of the state as soon as $q \geq p+1$, so that it is surrounded by at least $p$ unoccupied sites on both sides. This segment can now serve as a new unit cell with an energy density

$$
\frac{p E_{A}-E_{\Delta}}{p N / Q}=\frac{E_{A}}{N / Q}-\frac{E_{\Delta}}{p N / Q}
$$

which lowers the energy in contradiction to our assumptions. A similar process can be used to show that a ground state cannot have a sequence of $(p+2)$ or more unoccupied sites. Thus, we conclude that the largest spacing in any ground state has at most $p$ unoccupied sites.
Table III. Charge-ordered ground states (GS) and their energies in the atomic limit of systems with commensurable particle densities $Q=1 /(p-1)$, where $p$ is the range of the interactions. The degeneracy of these states is denoted as $f$, which accounts for the translational freedom and the possibility of a mirror-reflected phase. $L_{\max }$ is the maximal size of

\begin{tabular}{|c|c|c|}
\hline GS unit cell & Energy density & $f$ \\
\hline \multicolumn{3}{|c|}{$p=3, Q=1 / 2, L_{\max }=28$} \\
\hline$\bullet$ & $U_{2} / 2$ & 2 \\
\hline$\bullet \bullet \circ ০$ & $\left(U_{1}+U_{3}\right) / 4$ & 4 \\
\hline$\bullet \bullet \bullet \circ ০ ০$ & $\left(2 U_{1}+U_{2}\right) / 6$ & 6 \\
\hline \multicolumn{3}{|c|}{$p=4, Q=1 / 3, L_{\max }=36$} \\
\hline$\bullet \circ \circ$ & $U_{3} / 3$ & 3 \\
\hline$\bullet \bullet \circ ০ ০ ০$ & $U_{1} / 6$ & 6 \\
\hline$\bullet \bullet \bullet ০ ০ ০$ & $\left(U_{2}+U_{4}\right) / 6$ & 6 \\
\hline$\bullet \bullet \circ ০ 0 \bullet \bullet \circ ০$ & $\left(U_{1}+2 U_{4}\right) / 9$ & 9 \\
\hline$\bullet \bullet \bullet \circ \bullet ০ ০ ০ ০$ & $\left(2 U_{2}+U_{4}\right) / 9$ & 9 \\
\hline$\bullet \bullet \bullet ০ ০ \bullet ০ \bullet ০ ০ ০ ০$ & $\left(2 U_{2}+U_{3}\right) / 12$ & 12 \\
\hline$\bullet \bullet \bullet ০ ০ ০ ০ \bullet ০ \bullet ০ ০ ০ ০ \bullet ০ \bullet ০ ০ ০ ০$ & $\left(2 U_{1}+3 U_{2}\right) / 21$ & 21 \\
\hline \multicolumn{3}{|c|}{$p=5, Q=1 / 4, L_{\max }=32$} \\
\hline$\bullet \circ 00$ & $U_{4} / 4$ & 4 \\
\hline$\bullet \circ \circ \bullet \circ ০ ০ ০$ & $\left(U_{3}+U_{5}\right) / 8$ & 8 \\
\hline$\bullet \circ \bullet ૦ \circ)^{\prime}$ & $U_{2} / 8$ & 8 \\
\hline 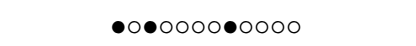 & $\left(U_{2}+2 U_{5}\right) / 12$ & 12 \\
\hline$\bullet \circ 0 \bullet \circ 0 \bullet \circ \circ 000$ & $2 U_{3} / 12$ & 12 \\
\hline$\bullet \bullet \circ ০ ০ 0 \bullet \bullet \circ ০ ০ ০ \bullet ০ ০ ০ ০$ & $\left(U_{1}+3 U_{5}\right) / 16$ & 16 \\
\hline$\bullet \bullet \circ 0000 \bullet \bullet \circ 0000 \bullet \bullet \circ 0000$ & $2 U_{1} / 20$ & 20 \\
\hline \multicolumn{3}{|c|}{$p=6, Q=1 / 5, L_{\max }=40$} \\
\hline$\bullet \circ 000$ & $U_{5} / 5$ & 5 \\
\hline$\bullet \circ ૦ 000 \bullet \circ \circ \circ$ & $\left(U_{4}+U_{6}\right) / 10$ & 10 \\
\hline$\bullet ० ০ 0000 \bullet ০ ০$ & $U_{3} / 10$ & 10 \\
\hline 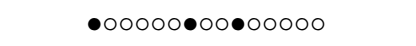 & $\left(U_{3}+2 U_{6}\right) / 15$ & 15 \\
\hline$\bullet ० ০ 0000 \bullet \bullet ০ ০ ০ ০ ০ ০$ & $U_{1} / 15$ & 15 \\
\hline$\bullet ० 00000 \bullet ० 00 \bullet \circ ० \circ$ & $2 U_{4} / 15$ & 15 \\
\hline 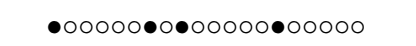 & $\left(U_{2}+3 U_{6}\right) / 20$ & 20 \\
\hline$\bullet \bullet ০ ০ ০ ০ \bullet \bullet ০ ০ ০ ০ ০ \bullet ০ ০ ০ ০ ০ \bullet ০ ০ ০ ০ ০$ & $\left(U_{1}+4 U_{6}\right) / 25$ & 25 \\
\hline$\bullet \bullet ০ ০ ০ ০ ০ \bullet \bullet \bullet ০ ০ ০ ০ ০ ০ \bullet \bullet \bullet ০ ০ ০ ০ ০ ০$ & $2 U_{2} / 25$ & 25 \\
\hline
\end{tabular}
the considered unit cell.

\section{Specific cases}

The general properties listed above allow us to significantly reduce the effective charge-configuration space of ground-state candidates. Based on Property 1 and exploiting the system's translational invariance, we can place the guaranteed large spacing towards the front of the sequence, and therefore fix the first $1 / Q$ sites to

$$
\bullet \underbrace{\circ 0 \cdots \circ}_{1 / Q-1} \text {. }
$$

This reduces the effective configuration space to reduced systems of size $(N-1) / Q$ and $(N-1)$ particles. Based on Property 2, we then can remove any charge configuration with unoccupied segments exceeding $p$, which at the same time significantly reduces the maximal unit-cell size encountered in the construction.

For each admissible state obtained in this way, we determined the general expression of the ground-state energy density as a function of the interaction parameters 
Table IV. Charge-ordered ground states as Table III but for systems with particle densities $Q=1 /(p-2)$.

\begin{tabular}{|c|c|c|}
\hline GS unit cell & Energy density & $\overline{f f}$ \\
\hline \multicolumn{3}{|c|}{$p=4, Q=1 / 2, L_{\max }=26$} \\
\hline$\bullet \circ$ & $\left(U_{2}+U_{4}\right) / 2$ & 2 \\
\hline$\bullet \bullet \circ \circ$ & $\left(U_{1}+U_{3}+2 U_{4}\right) / 4$ & 4 \\
\hline$\bullet \bullet \bullet \circ \circ ০$ & $\left(2 U_{1}+U_{2}+U_{4}\right) / 6$ & 6 \\
\hline$\bullet \bullet \bullet \bullet ० ০ ০ ০$ & $\left(3 U_{1}+2 U_{2}+U_{3}\right) / 8$ & 8 \\
\hline$\bullet \bullet \circ \bullet \circ \circ \bullet \circ$ & $\left(U_{1}+2 U_{2}+3 U_{3}\right) / 8$ & 8 \\
\hline \multicolumn{3}{|c|}{$p=5, Q=1 / 3, L_{\max }=27$} \\
\hline$\bullet \circ \circ$ & $U_{3} / 3$ & 3 \\
\hline$\bullet \circ \bullet \circ \circ \circ$ & $\left(U_{2}+U_{4}\right) / 6$ & 6 \\
\hline$\bullet \bullet \circ ০ ০ ০$ & $\left(U_{1}+U_{5}\right) / 6$ & 6 \\
\hline$\bullet \bullet \circ ০ ০ \bullet ০ ০ ০$ & $\left(U_{1}+2 U_{4}+2 U_{5}\right) / 9$ & 9 \\
\hline$\bullet \bullet \bullet \circ \bullet \bullet \circ ০ ০$ & $\left(2 U_{2}+U_{4}+U_{5}\right) / 9$ & 9 \\
\hline$\bullet \bullet \bullet \circ ০ \bullet \circ \bullet \circ ০ ০ ০$ & $\left(2 U_{2}+U_{3}+3 U_{5}\right) / 12$ & 12 \\
\hline$\bullet \bullet ০ ০ \bullet ০ ০ ০ \bullet ০ ০ ০ \bullet ০ ০$ & $\left(U_{1}+2 U_{3}+4 U_{4}\right) / 15$ & 15 \\
\hline$\bullet \bullet \bullet \circ ০ ০ ০ ০ \bullet \bullet \circ ০ ০ ০ ০$ & $\left(3 U_{1}+U_{2}\right) / 15$ & 15 \\
\hline$\bullet \bullet \circ ০ \bullet \bullet ০ ০ ০ ০ ০ \bullet \bullet ০ ০ ০ ০ ০$ & $\left(3 U_{1}+U_{3}+2 U_{4}+U_{5}\right) / 18$ & 18 \\
\hline$\bullet \bullet \circ ০ \bullet ০ ০ \bullet \bullet ০ ০ ০ ০ ০ \bullet \bullet ০ ০ ০ ০ ০$ & $\left(3 U_{1}+2 U_{3}+2 U_{4}\right) / 21$ & 21 \\
\hline$\bullet \bullet \bullet ০ ০ ০ ০ \bullet ০ \bullet ০ ০ ০ ০ \bullet ০ \bullet ০ ০ ০ ০$ & $\left(2 U_{1}+3 U_{2}+3 U_{5}\right) / 21$ & 21 \\
\hline$\bullet \bullet \bullet ০ ০ ০ ০ ০ \bullet \bullet \bullet ০ ০ ০ ০ ০ \bullet \bullet \bullet ০ ০ ০ ০ ০$ & $\left(4 U_{1}+3 U_{2}\right) / 24$ & 24 \\
\hline
\end{tabular}

Table V. Charge-ordered ground states as Table III but for systems at half filling $(Q=1 / 2)$.

\begin{tabular}{|c|c|c|}
\hline GS unit cell & Energy density & $\bar{f}$ \\
\hline \multicolumn{3}{|c|}{$p=5, Q=1 / 2, L_{\max }=26$} \\
\hline$\bullet \circ$ & $\left(U_{2}+U_{4}\right) / 2$ & 2 \\
\hline$\bullet \bullet \circ \circ$ & $\left(U_{1}+U_{3}+2 U_{4}+U_{5}\right) / 4$ & 4 \\
\hline$\bullet \bullet \bullet \bullet \circ ০$ & $\left(U_{1}+U_{2}+2 U_{3}+U_{4}+U_{5}\right) / 6$ & $2 \times 6$ \\
\hline$\bullet \bullet \bullet \circ \circ \circ$ & $\left(2 U_{1}+U_{2}+U_{4}+2 U_{5}\right) / 6$ & 6 \\
\hline$\bullet \bullet \circ \bullet ০ ০ \bullet ০$ & $\left(U_{1}+2 U_{2}+3 U_{3}+3 U_{5}\right) / 8$ & 8 \\
\hline$\bullet \bullet \bullet \bullet ০ ০ ০ ০$ & $\left(3 U_{1}+2 U_{2}+U_{3}+U_{5}\right) / 8$ & 8 \\
\hline$\bullet \bullet \circ \bullet \bullet \circ ০ \bullet ০ ০$ & $\left(2 U_{1}+U_{2}+4 U_{3}+3 U_{4}\right) / 10$ & 10 \\
\hline$\bullet \bullet \bullet \bullet \bullet ০ ০ ০ ০ ০$ & $\left(4 U_{1}+3 U_{2}+2 U_{3}+U_{4}\right) / 10$ & 10 \\
\hline \multicolumn{3}{|c|}{$p=6, Q=1 / 2, L_{\max }=26$} \\
\hline$\bullet$ & $\left(U_{2}+U_{4}+U_{6}\right) / 2$ & 2 \\
\hline$\bullet \bullet \circ \circ$ & $\left(U_{1}+U_{3}+2 U_{4}+U_{5}\right) / 4$ & 4 \\
\hline$\bullet \bullet \circ \bullet \circ \circ$ & $\left(U_{1}+U_{2}+2 U_{3}+U_{4}+U_{5}+3 U_{6}\right) / 6$ & $2 \times 6$ \\
\hline$\bullet \bullet \bullet \circ \circ ০$ & $\left(2 U_{1}+U_{2}+U_{4}+2 U_{5}+3 U_{6}\right) / 6$ & 6 \\
\hline$\bullet \circ \bullet \bullet \circ \bullet ০ ০$ & $\left(U_{1}+2 U_{2}+3 U_{3}+3 U_{5}+2 U_{6}\right) / 8$ & 8 \\
\hline$\bullet \bullet \bullet \bullet \circ ૦ \circ \circ$ & $\left(3 U_{1}+2 U_{2}+U_{3}+U_{5}+2 U_{6}\right) / 8$ & 8 \\
\hline$\bullet \bullet \circ \bullet \bullet \circ ০ \bullet \circ ০$ & $\left(2 U_{1}+U_{2}+4 U_{3}+3 U_{4}+3 U_{6}\right) / 10$ & 10 \\
\hline$\bullet \bullet \bullet \bullet \bullet ০ ০ ০ ০ ০$ & $\left(4 U_{1}+3 U_{2}+2 U_{3}+U_{4}+U_{6}\right) / 10$ & 10 \\
\hline$\bullet \circ \bullet \bullet \circ \bullet \circ \bullet \circ \circ \bullet \circ$ & $\left(U_{1}+4 U_{2}+3 U_{3}+2 U_{4}+5 U_{5}\right) / 12$ & 12 \\
\hline$\bullet \bullet \bullet \bullet \bullet \bullet \circ ০ ০ ০ ০ ০$ & $\left(5 U_{1}+4 U_{2}+3 U_{3}+2 U_{4}+U_{5}\right) / 12$ & 12 \\
\hline$\bullet \bullet \bullet \circ \bullet \circ \circ \circ \bullet \circ \bullet \bullet \bullet \circ \circ \bullet \circ \circ$ & $\left(4 U_{1}+4 U_{2}+4 U_{3}+5 U_{4}+2 U_{5}+3 U_{6}\right) / 18$ & 18 \\
\hline$\bullet \bullet \bullet \circ ০ ০ \bullet \bullet \circ \bullet \bullet ০ ০ \bullet ০ ০$ & $\left(4 U_{1}+3 U_{2}+5 U_{3}+5 U_{4}+2 U_{5}+3 U_{6}\right) / 18$ & $2 \times 18$ \\
\hline
\end{tabular}

$\left\{U_{m}\right\}$. Next, we discarded symbolically all configurations that can never drop below the energy densities of all other charge configurations. The final list contains the energy densities of all phases that can have the lowest energy for some set of values $\left\{U_{m}\right\}$. This leads to the charge-ordered phases listed in the following tables.

Table III lists the unit cells and energy densities for critical densities $Q=1 /(p-1)$ and $p=3,4,5,6$, with the unit-cell size limited to the specified values $L_{\max }$. In these cases we are highly confident that there are no ground states with larger unit cells. Table IV presents the unit cells and energy densities for $Q=1 /(p-2)$ with $p=4$ and 5 . Notice that for $p=5$ we find ground-state unit cells of size up to $\left(L_{\max }-3\right)$, so that we cannot fully exclude the possibility of additional ground-state configurations with even larger unit cells. Finally, results for $Q=1 / 2, p=5$ and 6 are presented in Table $\mathrm{V}$. Amongst the combinations listed in Table I, this leaves the case $p=6, Q=1 / 3$ where we find 63 phases with $L \leq L_{\max }=27$, and $p=6, Q=1 / 4$, where we find 23 phases with $L \leq L_{\max }=32$, which defines the limit of our computational capabilities; the corresponding phases 
are therefore not listed here.

In all these tables, the degeneracy of the states accounts for the translational displacement by a finite num- ber of sites (up to the size of the unit cell), as well as for the possible duplication by a distinct mirror-reflected phase.
* mszynisz@gmail.com

1 R. E. Peierls, Quantum Theory of Solids (Oxford University Press, London, 1955).

2 J. Voit, Rep. Prog. Phys. 58, 977 (1995).

3 S.-I. Tomonaga, Prog. Theor. Phys. 5, 544 (1950)

4 J. M. Luttinger, J. Math. Phys. 4, 1154 (1963).

5 D. C. Mattis and E. H. Lieb, J. Math. Phys. 6, 304 (1965)

${ }^{6}$ F. D. M. Haldane, J. Phys. C: Solid State Phys. 14, 2585 (1981)

F. D. M. Haldane, Phys. Rev. Lett. 47, 1840 (1981)

8 R. Peierls, Ann. Phys. (Berl.) 396, 121 (1930).

9 N. F. Mott and R. Peierls, Proc. Phys. Soc. 49, 72 (1937)

10 N. F. Mott, Proc. Phys. Soc. A 62, 416 (1949)

11 R. A. Bari, Phys. Rev. B 3, 2662 (1971)

12 G. Grüner, Rev. Mod. Phys. 60, 1129 (1988)

13 H. Fröhlich, Proc. R. Soc. Lond. Ser. A, Math. Phys. Sci. 223, 296 (1954)

${ }^{14}$ C. G. Kuper, Proc. R. Soc. Lond. Ser. A, Math. Phys. Sci. 227, 214 (1955)

15 J. Hubbard, Proc. R. Soc. Lond. A Math. Phys. Sci. 276, 238 (1963).

${ }^{10}$ B. Fourcade and G. Spronken, Phys. Rev. B 29, 5089 (1984)

${ }_{17}$ T. Giamarchi, Physica B 230-232, 975 (1997)

18 G. Gómez-Santos, Phys. Rev. Lett. 70, 3780 (1993).

19 P. Schmitteckert and R. Werner, Phys. Rev. B 69, 195115 (2004)

${ }^{20}$ T. Mishra, J. Carrasquilla, and M. Rigol, Phys. Rev. B 84, $115135(2011)$

${ }^{21}$ J. F. Dodaro, H.-C. Jiang, and S. A. Kivelson, Phys. Rev. B 95, 155116 (2017).

22 J. Voit, Phys. Rev. B 45, 4027 (1992)

${ }^{23}$ E. V. Tsiper and A. L. Efros, J. Phys.: Condens. Matter 9, L561 (1997).

${ }^{24}$ M. Nakamura, J. Phys. Soc. Jpn. 68, 3123 (1999)

25 A. K. Zhuravlev and M. I. Katsnelson, Phys. Rev. B 61, 15534 (2000).

${ }^{20}$ P. Sengupta, A. W. Sandvik, and D. K. Campbell, Phys. Rev. B 65, 155113 (2002)

2 C.-B. Duan and W.-Z. Wang, J. Phys.: Condens. Matter 23, 365602 (2011)

${ }^{28}$ R. A. Molina and P. Schmitteckert, Phys. Rev. B 75, 235104 (2007)

${ }^{29}$ K. Hallberg, E. Gagliano, and C. Balseiro, Phys. Rev. B 41, 9474 (1990)

so P. Jordan and E. Wigner, Z. Phys. 47, 631 (1928)

${ }^{31}$ E. Lieb, T. Schultz, and D. Mattis, Ann. Phys. 16, 407 (1961)

32 S. Katsura, Phys. Rev. 127, 1508 (1962)

33 F. D. M. Haldane, J. Phys. C: Solid State Phys. 12, 4791 (1979)

${ }^{34}$ M. P. A. Fisher, P. B. Weichman, G. Grinstein, and D. S. Fisher, Phys. Rev. B 40, 546 (1989).

35 M. Lubasch, V. Murg, U. Schneider, J. I. Cirac, and M.-C. Bañuls, Phys. Rev. Lett. 107, 165301 (2011)

${ }^{36}$ M. A. Cazalilla, R. Citro, T. Giamarchi, E. Orignac, and
M. Rigol, Rev. Mod. Phys. 83, 1405 (2011)

37 H. P. Büchler, G. Blatter, and W. Zwerger, Phys. Rev. Lett. 90, 130401 (2003).

38 X.-J. Liu, P. D. Drummond, and H. Hu, Phys. Rev. Lett. 94, 136406 (2005)

39 E. Haller, R. Hart, M. J. Mark, J. G. Danzl, L. Reichsöllner, M. Gustavsson, M. Dalmonte, G. Pupillo, and H.-C. Nägerl, Nature 466, 597 (2010)

40 S. R. White, Phys. Rev. Lett. 69, 2863 (1992)

41 S. R. White, Phys. Rev. B 48, 10345 (1993).

42 U. Schollwöck, Rev. Mod. Phys. 77, 259 (2005).

43 I. P. McCulloch, J. Stat. Mech: Theory Exp. 2007, P10014 (2007)

${ }^{44}$ I. P. McCulloch, (2007), arXiv:0804.2509 [cond-mat.strel].

45 U. Schollwöck, Ann. Phys. 326, 96 (2011)

46 I. P. McCulloch, "Matrix product toolkit," https:// people.smp.uq.edu.au/IanMcCulloch/mptoolkit/, version 1705 .

47 V. J. Emery and C. Noguera, Phys. Rev. Lett. 60, 631 (1988)

48 D. Poilblanc, S. Yunoki, S. Maekawa, and E. Dagotto, Phys. Rev. B 56, R1645 (1997)

49 R. Franco and A. A. Aligia, Phys. Rev. B 67, 172507 (2003)

50 H. Seo, K. Tsutsui, M. Ogata, and J. Merino, J. Phys. Soc. Jpn. 75, 114707 (2006)

${ }^{51}$ D. Perez-Garcia, F. Verstraete, M. M. Wolf, and J. I. Cirac, Quantum Inf. Comput. 7, 401 (2007)

52 F. Verstraete, V. Murg, and J. I. Cirac, Adv. Phys. 57, 143 (2008).

53 D. Perez-Garcia, F. Verstraete, M. M. Wolf, and J. I. Cirac, Quantum Inf. Comput. 8, 650 (2008)

54 U. Schollwöck, "DMRG: Ground States, Time Evolution, and Spectral Functions," in Emergent Phenomena in Correlated Matter (Modeling and Simulation, Vol. 3) edited by E. Pavarini, E. Koch, and U. Schollwöck (Verlag des Forschungszentrum Jülich, Jülich, Germany, 2013) Chap. 16.

55 C. Hubig, I. P. McCulloch, U. Schollwöck, and F. A. Wolf, Phys. Rev. B 91, 155115 (2015).

56 A. W. Sandvik, AIP Conf. Proc. 1297, 135 (2010).

57 G. Vidal, J. I. Latorre, E. Rico, and A. Kitaev, Phys. Rev. Lett. 90, $227902(2003)$

58 J. I. Latorre, E. Rico, and G. Vidal, Quantum Inf. Comput. 4, 48 (2004)

כy J. Eisert, M. Cramer, and M. B. Plenio, Rev. Mod. Phys. 82, 277 (2010)

कo P. Calabrese and J. Cardy, J. Stat. Mech: Theory Exp. 2004, P06002 (2004).

${ }^{61}$ L. Tagliacozzo, T. R. de Oliveira, S. Iblisdir, and J. I. Latorre, Phys. Rev. B 78, 024410 (2008)

${ }^{62}$ R. Nandkishore and D. A. Huse, Annu. Rev. Condens. Matter Phys. 6, 15 (2015)

${ }^{63}$ A. D. A. and P. Zlatko, Ann. Phys. (Berl.) 529, 1700169 (2017) 